\title{
Irreducible Unitary Representations of Quantum Lorentz Group
}

\author{
Wiesław Pusz \\ Department of Mathematical Methods in Physics, Faculty of Physics, University of Warsaw, \\ Hoza 74, PL 00-682 Warsaw, Poland
}

Received June 15, 1992

\begin{abstract}
A complete classification of irreducible unitary representations of a one parameter deformation $S_{q} L(2, C)(0<q<1)$ of $S L(2, C)$ is given. It shows that in spite of a popular belief the representation theory for $S_{q} L(2, C)$ is not "a smooth deformation" of the one for $S L(2, C)$.
\end{abstract}

\section{Introduction}

A theory of quantum deformations of the classical locally compact groups still seems far from being complete. According to [16] one can distinguish a purely algebraic (Hopf-algebra or Hopf *-algebra) level, topological ( $C^{*}$-algebra) level and intermediate Hilbert space (i.e. the representation theory) level. For the compact groups the algebraic and topological approaches are equivalent since the topological level is well understood and there is a natural way of passing to it from the algebraic one (see e.g. $[14,15]$ ) via the Hilbert space level. In effect one obtains a smooth deformation of group structure and its representation theory.

This experience is a source of the popular belief that it is also the case for general locally compact groups. A class of Pontryagin duals for compact quantum groups is also well established on the $C^{*}$-algebra level [8] and seems to confirm this conviction, but for the non-compact case there is no general theory of topological quantum deformation at the moment.

The study of other examples indicates that new phenomena can occur which are not seen on the algebraic level:

- The deformation may not exist on the $C^{*}$-algebra level (cf. [16] where nonexistence of comultiplication for quantum $S U(1,1)$ group for real values of deformation parameter was proved).

- The deformation on the $C^{*}$-algebra level exists under some additional conditions (e.g. restrictions on spectra of operators involved in the theory (see the spectral condition in [16] for the case of $E(2)$ - the group of motions of the Euclidean plane)). 
- The deformation exists on the $C^{*}$-algebra level but a representation theory is not similar to the undeformed one. To obtain a similarity one has to impose smoothness conditions (e.g. smooth and non-smooth finite-dimensional representations of the quantum Lorentz group in [8]).

It is clear that a study of concrete examples is very important for the proper background of the general theory. Investigating known examples one reveals a crucial role of a Hilbert space level. In the present paper we study irreducible unitary representations of the quantum Lorentz group [8]. This will be a starting point as well for the construction of Pontryagin dual (i.e. a quantum group of "characters") quantum Lorentz group on the $C^{*}$-algebra level as for the development of harmonic analysis in further investigations.

The quantum deformation $S_{q} L(2, C)(0<q<1)$ of $S_{1} L(2, C)=S L(2, C)$ introduced by Podleś and Woronowicz in [8] contains the quantum deformation $S_{q} U(2)$ of $S U(2)$. Any unitary representation $v$ of $S_{q} L(2, C)$ induces then a unitary representation $v_{c}$ of $S_{q} U(2)$. Since irreducible (unitary) representations of $S_{q} U(2)$ are labeled by the integer and half-integer spin parameter $s \in S$ where

$$
S=\left\{0, \frac{1}{2}, 1, \frac{3}{2}, 2, \frac{5}{2}, 3, \ldots\right\},
$$

let $p$ be a minimal spin occurring in the (unique) decomposition of $v_{c}$ into a direct sum of irreducible representations. Let for any $p \in S$,

$$
S_{p}=\{p, p+1, p+2, p+3, \ldots\}
$$

be a subset of $S$. By $\mathscr{E}_{p}$ we shall denote for $p \in S$ and $p \neq 0$ an ellipse in $C$ :

$$
\mathscr{E}_{p}=\left\{z: z=\frac{q}{\sqrt{1+q^{2}}}\left[\left(q^{p}+q^{-p}\right) \cos \varphi+i\left(q^{-p}-q^{p}\right) \sin \varphi\right] \text { for } \varphi \in[0,2 \pi[\}\right.
$$

and for $p=0$ a closed interval

$$
\mathscr{E}_{0}=\left[-\sqrt{1+q^{2}}, \sqrt{1+q^{2}}\right] .
$$

Let

$$
\Sigma_{q}=\bigcup_{p \in S} \mathscr{E}_{p} .
$$

Then we shall prove that for any irreducible unitary representation of $S_{q} L(2, C)$ the value $\mathscr{X}_{0}$ of the Casimir operator $X$ for $S_{q} L(2, C)$ belongs to $\Sigma_{q}$ and irreducible unitary representations can be labeled by the minimal spin $p$ and the value $\mathscr{X}_{0}$ analogously as in the case of classical $S L(2, C)$. Let

$$
\mathscr{L}_{q}=\left\{\left(p, z_{p}\right): p \in S, z_{p} \in \mathscr{E}_{p}\right\},
$$

then we can state our main result

Theorem 0.1. Let $q \in] 0,1\left[\right.$. There is one to one correspondence between the set $\mathscr{L}_{q}$ and the set of unitary equivalence classes of irreducible unitary representations of quantum Lorentz group $S_{q} L(2, C)$.

Moreover,

1. there are two one-dimensional representations:

$$
\tilde{\tau}=\left(0, \sqrt{1+q^{2}}\right) \text { and the trivial one } \tau=\left(0,-\sqrt{1+q^{2}}\right) .
$$


2. If $v$ is an unitary irreducible representation different from $\tau$ and $\tilde{\tau}$ with minimal spin $p$ then it is infinite-dimensional and contains any irreducible unitary representation of $S_{q} U(2)$ with spin $s \in S_{p}$ with multiplicity one.

To compare this result with the classical one (the case $q=1$ ) let us denote by $\mathscr{P}_{p}$ the parabola

$$
\mathscr{P}_{p}=\left\{z: z=2\left(t^{2}-p^{2}+1\right)+4 i p t \text { for } t \in\right]-\infty, \infty[\}
$$

for $p \in S$ and $p \neq 0$ and a halfline

$$
\mathscr{P}_{0}=[0, \infty[
$$

for $p=0$. Let

$$
\Sigma_{1}=\bigcup_{p \in S} \mathscr{P}_{p}
$$

and

$$
\mathscr{L}_{1}=\left\{\left(p, z_{p}\right): p \in S, z_{p} \in \mathscr{P}_{p}\right\} .
$$

Then the value $\mathscr{X}_{0}$ of the Casimir operator $X$ (a linear combination of the Laplace operators) for $S L(2, C)$ in the unitary irreducible representation $v$ of $S L(2, C)$ belongs to $\Sigma_{1}$ and the set of irreducible unitary representations (unitary equivalence classes) is labeled by $\mathscr{L}_{1}$ (see e.g. [5, pp. 104, 144] or [1, 2]). Fig. 1 describes the set $\frac{\sqrt{1+q^{2}}}{q} \Sigma_{q}$ for $0<q<1$ (upper part) and $\Sigma_{1}$ (lower
part). Let us note that

$$
\left\{\frac{\sqrt{1+q^{2}}}{q} \mathscr{E}_{p}: p \in S, p \neq 0\right\}
$$

is a family of cofocal ellipses with focuses in points $(-2,0)$ and $(2,0)$ and

$$
\left\{\mathscr{P}_{p}: p \in S, p \neq 0\right\}
$$

is a family of cofocal parabolas with focus in $(2,0)$

All label spaces $\mathscr{L}_{q}$ are homeomorphic for $0<q<1$ and not homeomorphic with $\mathscr{L}_{1}$. The limit $q \rightarrow 1$ is singular. It corresponds to moving one focus to infinity and then representation $\tilde{\tau}$ disappears (there are also two complementary series in the deformed case and one of them also disappears in this limit).

Let us observe that if $v$ is an irreducible unitary representation of quantum Lorentz group then $\tilde{\tau} \otimes v$ is also an irreducible unitary representation [this corresponds to the reflection $\Sigma_{q}$ with respect to the point $\left.(0,0)\right]$.

In essence it was also noticed in the case of irreducible finite-dimensional (nonunitary) representations of $S_{q} L(2, C)$ [8]. Since in this case the set of values of Casimir operator is discrete it was possible to divide representations into two parts - the smooth representations which are continuous deformations of representations for $q=1$ and non-smooth one. It was conjectured [8] that this exhausts all finite-dimensional irreducible representations of $S_{q} L(2, C)$. The affirmative answer was given in [12].

Such a division is no longer possible in the case of unitary representations and this shows that also in the case of finite-dimensional representations the nonsmooth ones are also important. 


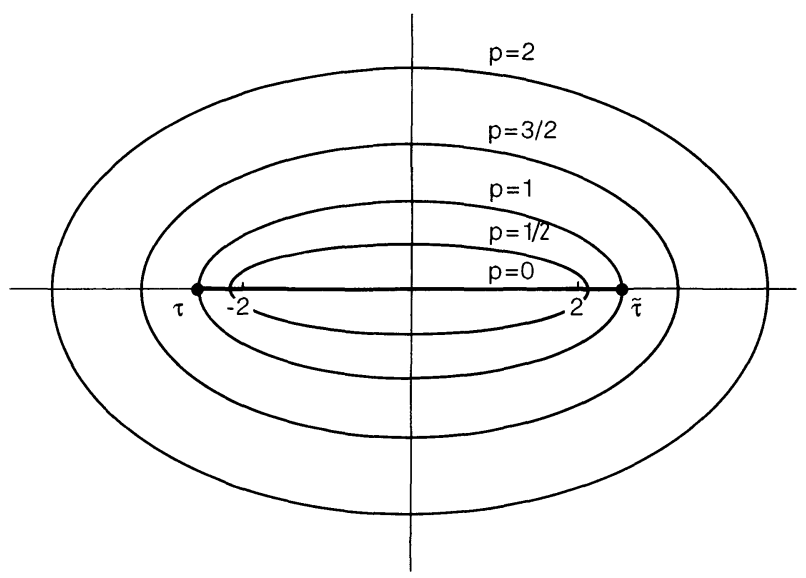

Fig. 1. $\Sigma_{q}$

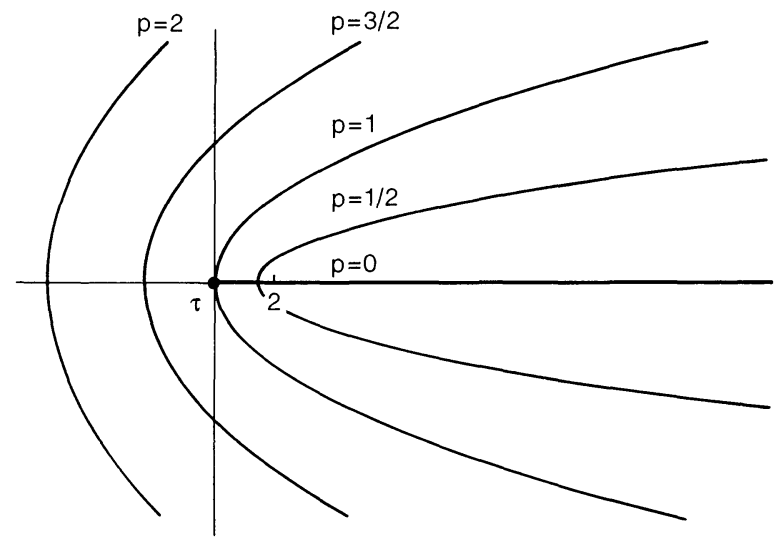

A few remarks on notations: For any $C^{*}$-algebra $B$ by $M(B)$ we shall denote the multiplier algebra of $B$. Let us note that for the algebra of compact operators $C B(H)$ on a Hilbert space $H$ we have that $M(C B(H))=B(H)$. We shall say that $\psi \in \operatorname{Mor}\left(B_{1}, B_{2}\right)$ if $\psi$ is *-algebra homomorphism $\psi: B_{1} \rightarrow M\left(B_{2}\right)$ such that $\psi\left(B_{1}\right) B_{2}$ is dense in $B_{2}$ (see [16, p. 402]).

We shall also use an affiliation relation in the case of "unbounded multiplier" $T$ and denote it $T \eta B$ (cf. $[16, \S 1]$ ). Then any $\psi \in \operatorname{Mor}\left(B_{1}, B_{2}\right)$ has a unique extension to *-algebra homomorphism from $M\left(B_{1}\right)$ to $M\left(B_{2}\right)$ and also to elements affiliated with $B_{1}$ [16, Theorem 1.2].

Let $\left\{B_{n}\right\}_{n \in N}$ be a family of finite-dimensional (unital) $C^{*}$-algebras labeled by a denumerable set $N$. Then

$$
B=\sum_{n \in N}^{\oplus} B_{n}
$$

will denote the (non-unital) $C^{*}$-algebra which elements are sequences $\left(b_{n}\right)_{n \in N}$ tending to 0 at infinity. In this case

$$
M(B)=\sum_{n \in N}^{\text {bounded }} B_{n},
$$


i.e. $b \in M(B)$ if $b=\left(b_{n}\right)_{n \in N}$ is a uniformly bounded sequence and $b \eta B$ if $b \in \underset{n \in N}{X_{n}} B_{n}$ $\left[16, \S 1\right.$, Example 5]. By $\sum_{n \in N}^{\text {finite }} B_{n}$ we shall denote a dense *-algebra in $B$ of sequences $\left(b_{n}\right)_{n \in N}$ with finite number of elements different from 0 .

In the paper we deal with compact as well as non-compact quantum groups. In any case quantum group $G$ is a bialgebra with additional "group structure". A bialgebra is a pair $(\mathfrak{R}, \Delta)$, where $\mathfrak{R}$ is a $C^{*}$-algebra and $\Delta \in \operatorname{Mor}(\mathfrak{R}, \mathfrak{R} \otimes \mathfrak{R})$ is a coassociative comultiplication:

$$
(\Delta \otimes \mathrm{id}) \Delta=(\mathrm{id} \otimes \Delta) \Delta .
$$

We shall say that $v \in M(C B(H) \otimes \Re)$ is a strongly continuous representation of $G=(\Re, \Delta)$ (acting) on the Hilbert space $H$ if

$$
\left(\mathrm{id}_{H} \otimes \Delta\right) v=v_{12} v_{13} .
$$

[We use the leg notation: for any $C^{*}$-algebras $A, B, C$ and $w \in M(A \otimes B)$ by $w_{12}$ we denote the unique image of $w$ under the canonical morphism

$$
\psi_{12} \in \operatorname{Mor}(A \otimes B \otimes C),
$$

where

$$
\psi_{12}(a \otimes b)=a \otimes b \otimes I_{C} \in \mathrm{M}(A \otimes B \otimes C)
$$

for any $a \in A, b \in B$, etc.] We shall say that the representation is unitary if element $v$ is unitary.

Any quantum group $G=(\Re, \Delta)$ considered in this paper is a one parameter deformation of a classical object with deformation parameter $q \in] 0,1[$ and has a 2-dimensional fundamental representation

$$
u=\left(\begin{array}{ll}
u_{11} & u_{12} \\
u_{21} & u_{22}
\end{array}\right) .
$$

Then the algebra $\mathfrak{R}$ is "generated" by $\left\{u_{i j}, u_{i j}^{*}\right\}$ satisfying some commutation relations. The multiplication $\Delta$ is then deduced from its value on the sets of generators

$$
\Delta\left(u_{i j}\right)=u_{i 1} \otimes u_{1 j}+u_{i 2} \otimes u_{2 j} .
$$

There is also a counit $e$ and coinverse (antipode) $\kappa$ which can be "extended" from their values on generators:

$$
\begin{gathered}
e\left(u_{i j}\right)=\delta_{i j}=e\left(u_{i j}^{*}\right), \\
\kappa\left(u_{11}\right)=u_{22}, \quad \kappa\left(u_{11}^{*}\right)=u_{22}^{*}, \\
\kappa\left(u_{22}\right)=u_{11}, \quad \kappa\left(u_{22}^{*}\right)=u_{11}^{*}, \\
\kappa\left(u_{12}\right)=-\frac{1}{q} u_{12}, \quad \kappa\left(u_{12}^{*}\right)=-q u_{12}^{*}, \\
\kappa\left(u_{21}\right)=-q u_{21}, \quad \kappa\left(u_{21}^{*}\right)=-\frac{1}{q} u_{21}^{*} .
\end{gathered}
$$

For the precise meaning of "generating" and "extension" in concrete examples we refer the reader to [8]. 
The paper is organized as follows. The first section is selfcontained. It embodies the idea that the Pontryagin dual quantum group (the universal "space of unitary characters" for quantum group) play the same role in the representation theory of quantum group as the Lie algebra in the Lie group theory. One has to replace derivatives by suitable functionals. This reduces the problem of study of the unitary representations to the problem (easier in general) of representations of the "algebra of functions" on the dual group.

Propositions 1.1 and 1.3 states that $S_{q} U(2)$ and $S_{q} \hat{U}(2)$ are mutually Pontryagin dual groups. This combining with the structural theorem for $S_{q} L(2, C)$ leads to the description of the algebra of "functions" on the dual group (Propositions 1.4 and 1.5).

To establish a useful framework for the study of representations of this algebra we develop the method of tensor operators in Sect. 2. Using this we are able to classify the unitary representations of $S_{q} L(2, C)$ (Propositions 3.1 and 3.2) and to realize unitary equivalence classes (Theorem 3.3) in the similar way as for the classical $S L(2, C)$.

\section{Quantum Lorentz Group $S_{q} L(2, C)$}

Recently, it was shown that the classical group $S L(2, C)$ admits many oneparameter non-equivalent deformations (see $[8,18,19])$. In this paper we focus on the first known.

For $q \in] 0,1$ [ we denote by $S_{q} L(2, C)$ the quantum deformation of $S L(2, C)$ in the sense of Podleś and Woronowicz [8]. This deformation follows from an analog of the Iwasawa decomposition for $S L(2, C)$ and it contains the well-known quantum deformation $S_{q} U(2)$ of $S U(2)$ and its Pontryagin dual $S_{q} \widehat{U}(2)$ in such a way that $S_{q} L(2, C)$ is an example of double-group construction applied to $S_{q} U(2)$. Symbolically,

$$
S_{q} L(2, C)=S_{q} U(2) \triangleright \triangleleft S_{q} \widehat{U}(2) .
$$

Since our description of unitary representations of $S_{q} L(2, C)$ is based upon this decomposition we recall in the first part of this section the basic results concerning $S_{q} U(2)$ and its representation theory. For more information we refer to $[13,8$, 11, 3].

To abbreviate the notation the compact quantum group $S_{q} U(2)$ will be denoted by $G_{c}$, its dual $S_{q} \hat{U}(2)$ (quantum non-compact group) by $G_{d}$ and by $G=G_{c} \bowtie G_{d}$ the resulting quantum (non-compact) Lorentz group $S_{q} L(2, C)$.

In the case of $G_{c}=\left(\Re_{c}, \Delta_{c}\right)$ the algebra $\mathfrak{R}_{c}$ of "continuous functions" on $G_{c}$ is the $C^{*}$-algebra completion of the $*$-algebra $\mathscr{A}_{c}$ of "smooth continuous functions" on $G_{c}$, i.e. the $*$-algebra generated by two elements $\alpha_{c}, \gamma_{c}$ such that

$$
\begin{gathered}
\alpha_{c}^{*} \alpha_{c}+\gamma_{c}^{*} \gamma_{c}=I, \quad \alpha_{c} \alpha_{c}^{*}+q^{2} \gamma_{c}^{*} \gamma_{c}=I, \\
\alpha_{c} \gamma_{c}=q \gamma_{c} \alpha_{c}, \quad \alpha_{c} \gamma_{c}^{*}=q \gamma_{c}^{*} \alpha_{c}, \quad \gamma_{c} \gamma_{c}^{*}=\gamma_{c}^{*} \gamma_{c} .
\end{gathered}
$$

To describe a group structure on $G_{c}$ it is enough to define it on the set of its generators. The comultiplication $\Delta_{c} \in \operatorname{Mor}\left(\mathfrak{R}_{c}, \mathfrak{R}_{c} \otimes \mathfrak{R}_{c}\right)$ follows from the fact that

$$
u^{1 / 2}=\left(\begin{array}{cc}
\alpha_{c} & -q \gamma_{c}^{*} \\
\gamma_{c} & \alpha_{c}^{*}
\end{array}\right)
$$


is a 2-dimensional (unitary) representation of $G_{c}$, i.e.

$$
\begin{aligned}
& \Delta_{c}\left(\alpha_{c}\right)=\alpha_{c} \otimes \alpha_{c}-q \gamma_{c}^{*} \otimes \gamma_{c}, \\
& \Delta_{c}\left(\gamma_{c}\right)=\gamma_{c} \otimes \alpha_{c}+\alpha_{c}^{*} \otimes \gamma_{c} .
\end{aligned}
$$

Moreover, there exists a counit $e_{c} \in \operatorname{Mor}\left(\Re_{c}, C\right)$ given by

$$
e_{c}\left(\alpha_{c}\right)=1, \quad e_{c}\left(\gamma_{c}\right)=0 .
$$

It is known that unitary irreducible representations of $G_{c}$ are labeled by spin parameter $s \in S$. The corresponding unitary representation $u^{s}$ acts on $(2 s+1)$ dimensional Hilbert space $K^{s}$ and $u^{s} \in B\left(K^{s}\right) \otimes \mathscr{A}_{c}$. The algebra $\mathfrak{R}_{d}$ of "continuous functions tending to 0 at infinity" on $G_{d}$ is then the $C^{*}$-algebra

$$
\mathfrak{R}_{d}=\sum_{s \in S}^{\oplus} B\left(K^{s}\right),
$$

i.e. the algebra without unity which means that we deal with non-compact case. The algebra $\Re_{d}$ can be generated by "unbounded continuous functions" $A_{d}$ and $N_{d}$ and then we have only $A_{d} \eta \Re_{d}, N_{d} \eta \Re_{d}$ (cf. [16, Sect. 1, Example 5]. These operators satisfy the following relations (cf. $[8,(1.35)-(1.38)]$ ):

$A_{d}$ is positive selfadjoint,

$$
\begin{aligned}
& A_{d} N_{d}=q N_{d} A_{d}, \\
& N_{d} N_{d}^{*}=N_{d}^{*} N_{d}+\frac{1}{1-q^{2}}\left(A_{d}^{-2}-A_{d}^{2}\right) .
\end{aligned}
$$

Let us note that $N_{d}$ differs from $n$ in [8] by the factor $\left(1-q^{2}\right)$. The comultiplication $\Delta_{d} \in \operatorname{Mor}\left(\mathfrak{R}_{d}, \mathfrak{R}_{d} \otimes \mathfrak{R}_{d}\right)$ on $G_{d}=\left(\mathfrak{R}_{d}, \Delta_{d}\right)$ is defined on generators $A_{d}, N_{d}$ by the fact that (cf. $[8$, Theorem 5.1])

$$
w_{d}=\left(\begin{array}{cc}
A_{d} & \left(1-q^{2}\right) N_{d} \\
0 & A_{d}^{-1}
\end{array}\right)
$$

is a 2-dimensional representation of $G_{d}$, i.e.

$$
\begin{aligned}
& \Delta_{d}\left(A_{d}\right)=A_{d} \otimes A_{d}, \\
& \Delta_{d}\left(N_{d}\right)=A_{d} \otimes N_{d}+N_{d} \otimes A_{d}^{-1} .
\end{aligned}
$$

Moreover, there exists a counit $e_{d} \in \operatorname{Mor}\left(\Re_{d}, C\right)$ defined by

$$
e_{d}\left(A_{d}\right)=1, \quad e_{d}\left(N_{d}\right)=0 .
$$

The fundamental role in the $G_{c}$-representation theory plays an unitary operator

$$
U=\sum_{s \in S}^{\text {bounded }} u^{s} .
$$

Clearly, $U \in M\left(\Re_{d} \otimes \Re_{c}\right)$. Since (cf. [8, (2.15) and Theorem 3.1 (3.3)])

$$
\left(\mathrm{id}_{d} \otimes \Delta_{c}\right) U=U_{12} U_{13}
$$

and

$$
\left(\Delta_{d} \otimes \mathrm{id}_{c}\right) U^{*}=U_{13}^{*} U_{23}^{*}
$$


it is called a bicharacter ( $U$ is a representation of $G_{c}$ acting on $\Re_{d}$ and $U^{*}$ is a representation of $G_{d}$ acting on $\mathfrak{R}_{c}$ ). This gives a correspondence between the unitary representations of a group and the representations of an "algebra of functions" on the Pontryagin dual group.

Proposition 1.1. i) Let $v_{c} \in M\left(C B(H) \otimes \Re_{c}\right)$ be a unitary representation of $G_{c}$ acting on Hilbert space $H$. Then there exists the unique $\psi_{d} \in \operatorname{Mor}\left(\Re_{d}, C B(H)\right)$ such that

$$
v_{c}=\left(\psi_{d} \otimes \mathrm{id}_{c}\right) U
$$

ii) Let $\psi_{d} \in \operatorname{Mor}\left(\Re_{d}, C B(H)\right)$. Then $v_{c}$ defined by (1.14) belongs to $M\left(C B(H) \otimes \Re_{c}\right)$ and it is a unitary representation of $G_{c}$.

Proof. The first part of the proposition follows from the more general Theorem 2.1 [8] in the special case of the $C^{*}$-algebra $C B(H)$. Since $U$ is the unitary operator, the unitarity of $v_{c}$ in (1.14) is obvious and by simple calculations using (1.12) we get that $v_{c}$ is the unitary representation of $G_{c}$ and ii) follows. Q.E.D.

Now we shall describe the correspondence $v_{c} \rightarrow \psi_{d}$ in a more convenient way, i.e. in terms of

$$
A=\psi_{d}\left(A_{d}\right), \quad N=\psi_{d}\left(N_{d}\right) .
$$

To this end let's define linear functionals $\varphi_{A}, \varphi_{R}$, and $\varphi_{N}$ on the algebra $\mathscr{A}_{c}$ such that for any $a, b \in \mathscr{A}_{c}$,

$$
\begin{gathered}
\varphi_{A}(a b)=\varphi_{A}(a) \varphi_{A}(b), \quad \varphi_{R}(a b)=\varphi_{R}(a) \varphi_{R}(b), \\
\varphi_{N}(a b)=\varphi_{A}(a) \varphi_{N}(b)+\varphi_{N}(a) \varphi_{R}(b) .
\end{gathered}
$$

They are uniquely defined by their values on the set of generators $\left\{\alpha, \alpha^{*}, \gamma, \gamma^{*}\right\}$ of the algebra $\mathscr{A}_{c}$ (cf. [8, Eqs. (5.1), (5.2)] and [9]):

$$
\begin{array}{cc}
\varphi_{A}\left(\alpha_{c}\right)=q^{1 / 2}=\varphi_{R}\left(\alpha_{c}^{*}\right), & \varphi_{A}\left(\alpha_{c}^{*}\right)=q^{-1 / 2}=\varphi_{R}\left(\alpha_{c}\right), \\
\varphi_{N}(\gamma)=-q^{-3 / 2}, & \varphi_{A}\left(I_{c}\right)=1=\varphi_{R}\left(I_{c}\right),
\end{array}
$$

and the other values are 0 . (Let us note that our $\varphi_{N}$ differs by the factor $\left(1-q^{2}\right)$ from $\xi_{12}$ in [8].)

Since any element $T \eta \mathfrak{R}_{d}=\sum_{s \in S}^{\oplus} B\left(K^{s}\right)$ is a sequence $\left(T_{s}\right)_{s \in S}$, where $T_{s} \in B\left(K^{s}\right)$ we have that

$$
A_{d}=\left(A_{s}\right)_{s \in S}, \quad N_{d}=\left(N_{s}\right)_{s \in S} .
$$

It was shown (see the proof of Theorem 5.1 in [8]) that for $s \in S$,

$$
\left(\operatorname{id}_{K^{s}} \otimes \varphi_{A}\right)\left(u^{s}\right)^{*}=A_{s}, \quad\left(\operatorname{id}_{K^{s}} \otimes \varphi_{N}\right)\left(u^{s}\right)^{*}=N_{s}, \quad\left(\operatorname{id}_{K^{s}} \otimes \varphi_{R}\right)\left(u^{s}\right)^{*}=A_{s}^{-1} .
$$

We shall shortly write this as

$$
A_{d}=\left(\mathrm{id}_{d} \otimes \varphi_{A}\right) U^{*}, \quad N_{d}=\left(\mathrm{id}_{d} \otimes \varphi_{N}\right) U^{*}, \quad A_{d}^{-1}=\left(\mathrm{id}_{d} \otimes \varphi_{R}\right) U^{*} .
$$

$A_{s}, N_{s}$ belong to $B\left(K^{s}\right)$ and satisfy relations (1.7) so they define the representation of $C^{*}$ - algebra $\mathfrak{R}_{d}$ which corresponds to the unitary representation $u^{s}$ of $G_{c}$. Moreover (cf. [8, Corollary 5.2]), there is the canonical orthonormal basis

$$
\left\{f_{m}^{s}: m=-s,-s+1, \ldots, s-1, s\right\}
$$


in $K^{s}$ such that

$$
\begin{aligned}
& A_{s} f_{m}^{s}=q^{m} f_{m}^{s}, \\
& N_{s} f_{m}^{s}=q^{-s} \sqrt{[s-m]_{q}[s+m+1]_{q}} f_{m+1}^{s}, \\
& N_{s}^{*} f_{m}^{s}=q^{-s} \sqrt{[s+m]_{q}[s-m+1]_{q}} f_{m-1}^{s},
\end{aligned}
$$

where

$$
[n]_{q}=\frac{1-q^{2 n}}{1-q^{2}} .
$$

Let us note that the canonical basis (1.19) is determined by (1.20) up to $m$-independent complex number of modulus 1 and can be fixed by identification of $K^{s}$ with the subspace of the $q$-symmetric tensors in $\bigotimes_{j=1}^{2 s} K^{1 / 2}$. Then $f_{s}^{s}=\bigotimes_{j=1}^{2 s} f_{1 / 2}^{1 / 2}$. For $u^{1 / 2}$ (in this case $K^{1 / 2}=C^{2}$ ) we have by $[8,(5.8)]^{j=1}$ the correspondence

$$
f_{-1 / 2}^{1 / 2}=\left(\begin{array}{l}
1 \\
0
\end{array}\right), \quad f_{1 / 2}^{1 / 2}=\left(\begin{array}{l}
0 \\
1
\end{array}\right),
$$

and then for $s=1$,

$$
\begin{gathered}
f_{1}^{1}=f_{1 / 2}^{1 / 2} \otimes f_{1 / 2}^{1 / 2}, \quad f_{-1}^{1}=f_{-1 / 2}^{1 / 2} \otimes f_{-1 / 2}^{1 / 2}, \\
f_{0}^{1}=\frac{1}{\sqrt{1+q^{2}}}\left[q f_{-1 / 2}^{1 / 2} \otimes f_{1 / 2}^{1 / 2}+f_{1 / 2}^{1 / 2} \otimes f_{-1 / 2}^{1 / 2}\right] .
\end{gathered}
$$

Now let $\psi_{d} \in \operatorname{Mor}\left(\Re_{d}, C B(H)\right)$ and $v_{c}$ be the corresponding unitary representation of $G_{c}$ acting on $H$. Since $\mathfrak{R}_{d}$ is a direct sum of full matrix algebras then

$$
H=\sum_{s \in S}^{\oplus} H^{s}=\sum_{s \in S}^{\oplus} K^{s} \otimes H_{s}
$$

and $\psi_{d}\left(a_{s}\right)=a_{s} \otimes \mathrm{id}_{H_{s}} \in B\left(H^{s}\right)$ for any $a_{s}$ belonging to the subalgebra $B\left(K^{s}\right)$ of $\mathfrak{R}_{d}$. The decomposition (1.22) corresponds to the decomposition of $v_{c}$ into a direct sum of irreducible unitary representations of $G_{c}$ and $v_{c}$ restricted to $H^{s}$ is $u^{s} \otimes \mathrm{id}_{H_{s}}$. The dimension $\operatorname{dim} H_{s}$ is a multiplicity of $u^{s}$ in $v_{c}$ [or equivalently $B\left(K^{s}\right)$ in $\psi_{d}$ ]. Let

$$
S\left(v_{c}\right):=\left\{s \in S: H^{s} \neq 0\right\}
$$

and

$$
D=\sum_{s \in S\left(v_{c}\right)}^{\text {finite }} H^{s}
$$

Then $S\left(v_{c}\right)$ will be called a support of $v_{c}$ (or a support of $\psi_{d}$ ) and $D$ will be called the natural domain for $v_{c}$ (and $\psi_{d}$ ) since it is a dense linear subset of $H$ invariant under the action of $v_{c}$ and an invariant essential domain for a selfadjoint operator

$$
A=\psi_{d}\left(A_{d}\right)=\sum_{s \in S\left(v_{c}\right)} A_{s} \otimes \mathrm{id}_{H_{s}}
$$

and closed operator

$$
N=\psi_{d}\left(N_{d}\right)=\sum_{s \in S\left(v_{c}\right)} N_{s} \otimes \mathrm{id}_{H_{s}},
$$


and hence for its adjoint $N^{*}$. It is clear that $A, N$, and $N^{*}$ satisfy (1.7) on $D$ and completely determine $\psi_{d}$. Now let $x \in H^{s}$, then $v_{c}\left(x \otimes I_{c}\right) \in D \otimes_{\mathrm{alg}} \mathscr{A}_{c}$ and

$$
\begin{aligned}
A x & =\psi_{d}\left(A_{d}\right) x=\left(\psi_{d} \otimes \mathrm{id}_{c}\right)\left[\left(\mathrm{id}_{d} \otimes \varphi_{A}\right) U^{*}\right] x \\
& =\left(\psi_{d} \otimes \varphi_{A}\right) U^{*}\left(x \otimes I_{c}\right)=\left(\mathrm{id}_{D} \otimes \varphi_{A}\right) v_{c}^{*}\left(x \otimes I_{c}\right) .
\end{aligned}
$$

In the same manner we can compute $N x$ and we get

Corollary 1.2. Let $v_{c} \in M\left(C B(H) \otimes \Re_{c}\right)$ be a unitary representation of $G_{c}$ acting on the Hilbert space $H, \psi_{d} \in \operatorname{Mor}\left(\mathfrak{R}_{d}, C B(H)\right)$ be the corresponding representation of $\mathfrak{R}_{d}$ and $D$ be the natural domain for $v_{c}$. Let $A=\psi_{d}\left(A_{d}\right), N=\psi_{d}\left(N_{d}\right)$.

Then for any $x \in D$,

$$
\begin{aligned}
& A x=\left(\mathrm{id}_{D} \otimes \varphi_{A}\right) v_{c}^{*}\left(x \otimes I_{c}\right), \\
& N x=\left(\mathrm{id}_{D} \otimes \varphi_{N}\right) v_{c}^{*}\left(x \otimes I_{c}\right) .
\end{aligned}
$$

Remark. This shows that in the sense of operators on $D$ we have equality

$$
A=\left(\mathrm{id}_{D} \otimes \varphi_{A}\right) v_{c}^{*}, \quad N=\left(\mathrm{id}_{D} \otimes \varphi_{N}\right) v_{c}^{*} .
$$

Let us note that using unitarity of $v_{c}$ and property (1.15) of $\varphi_{A}, \varphi_{N}$, and $\varphi_{R}$ we can also compute that

$$
\left(\mathrm{id}_{D} \otimes \varphi_{A}\right) v_{c}=A^{-1}, \quad\left(\mathrm{id}_{D} \otimes \varphi_{R}\right) v_{c}=A, \quad\left(\mathrm{id}_{D} \otimes \varphi_{N}\right) v_{c}=-\frac{1}{q} N
$$

as for operators on $D$. Let $\varphi^{*}(a)=\overline{\varphi\left(a^{*}\right)}$ for any $\varphi \in \mathscr{A}_{c}^{\prime}$ and $a \in \mathscr{A}_{c}$ then $\varphi^{*} \in \mathscr{A}_{c}^{\prime}$. For $\varphi_{N^{*}}:=\varphi_{N}^{*}$ we get

$$
N^{*}=\left(\mathrm{id}_{D} \otimes \varphi_{N^{*}}\right) v_{c} .
$$

Since [cf. (1.16)] $\varphi_{A}^{*}=\varphi_{R}, \varphi_{R}^{*}=\varphi_{A}$ we have by (1.15) that

$$
\varphi_{N^{*}}(a b)=\varphi_{A}(a) \varphi_{N^{*}}(b)+\varphi_{N^{*}}(a) \varphi_{R}(b),
$$

and using this

$$
\left(\mathrm{id}_{D} \otimes \varphi_{N^{*}}\right) v_{c}^{*}=-\frac{1}{q} N^{*}
$$

Now we shall prove that $G_{c}=S_{q} U(2)$ is a Pontryagin dual to $G_{d}$, i.e. $\widehat{G}_{d}=G_{c}$. This statement is not true for general compact quantum group and is closely related to the fact that the Haar measure $h_{c}$ may not be faithful on the whole $\mathfrak{R}_{c}$ but this does not occur in the case of $S_{q} U(2)$.

Proposition 1.3. i) Let $v_{d} \in M\left(C B(H) \otimes \Re_{d}\right)$ be a unitary representation of $G_{d}$ acting on Hilbert space $H$.

Then there exists the unique $\psi_{c} \in \operatorname{Mor}\left(\Re_{c}, C B(H)\right)$ such that

$$
v_{d}=\tau_{d}\left(\mathrm{id}_{d} \otimes \psi_{c}\right) U^{*},
$$

where $\tau_{d}$ is a flip $\tau_{d}: \mathfrak{R}_{d} \otimes B(H) \rightarrow B(H) \otimes \mathfrak{R}_{d}$.

ii) Let $\psi_{c} \in \operatorname{Mor}\left(\Re_{c}, C B(H)\right)$.

Then $v_{d}$ defined by (1.32) belongs to $M\left(C B(H) \otimes \mathfrak{R}_{d}\right)$ and is a unitary representation of $G_{d}$. 
Proof. The unitarity of $U^{*}$ implies that the operator $v_{d}$ defined by (1.32) is unitary and simple calculation using the bicharacter property (1.13) shows that it is a representation of $G_{d}$.

Now we shall prove i). Let $v_{d}$ be a unitary element of

$$
M\left(C B(H) \otimes \Re_{d}\right)=M\left(\sum_{s \in S}^{\oplus} C B(H) \otimes B\left(K^{s}\right)\right)=\sum_{s \in S}^{\text {bounded }} B(H) \otimes B\left(K^{s}\right)
$$

and $\pi_{s} \in \operatorname{Mor}\left(\Re_{d}, B\left(K^{s}\right)\right)$ be the canonical projection then

$$
v_{d}=\sum_{s \in S}^{\text {bounded }} v^{s},
$$

where $v^{s}=\left(\mathrm{id}_{H} \otimes \pi_{s}\right) v_{d}$. Choosing for any $s \in S$ the canonical orthonormal basis (1.19) in $K^{s}$ we get the isomorphism $B\left(K^{s}\right) \sim M_{2 s+1}(C)$. Let $\left\{m_{i j}^{s}\right\}$ be the matrix units for $M_{2 s+1}(C)$ then $v^{s} \in M_{2 s+1}(B(H))$ and

$$
\left(v^{s}\right)^{*}=\sum_{i, j} x_{i j}^{s} \otimes m_{i j}^{s}
$$

for some $x_{i j}^{s} \in B(H)$. Analogously, for $U=\sum_{s \in S}^{\text {bounded }} u^{s}$ we get

$$
u^{s}=\sum_{i, j} m_{i j}^{s} \otimes a_{i j}^{s},
$$

where $a_{i j}^{s} \in \mathscr{A}_{c}$ are matrix elements of $u^{s}$. Since the matrix elements of the irreducible unitary representations of $G_{c}$ form a linear basis of the vector space $\mathscr{A}_{c}$ $\left[14\right.$, Theorem 5.7(1)] then the map $\psi_{c}: \mathscr{A}_{c} \rightarrow B(H)$ defined by

$$
\psi_{c}\left(a_{i j}^{s}\right)=x_{i j}^{s}
$$

is linear. We have to prove that if $v_{d}$ is a representation of $G_{d}$ then $\psi_{c}$ is a multiplicative and $*$-preserving map. The multiplicativity of $\psi_{c}$ follows from the fact that

$$
\left(\Delta_{d} \otimes \mathrm{id}_{c}\right) U=U_{23} U_{13}=\sum_{s, s^{\prime}, i, j, i^{\prime}, j^{\prime}} m_{i j}^{s} \otimes m_{i^{\prime} j^{\prime}}^{s^{\prime}} \otimes a_{i^{\prime} j^{\prime}}^{s^{\prime}} a_{i j}^{s}
$$

and

$$
\left(\mathrm{id}_{H} \otimes \Delta_{d}\right) v_{d}^{*}=\left(v_{d}\right)_{13}^{*}\left(v_{d}\right)_{12}^{*}=\sum_{s, s^{\prime}, i, j, i^{\prime}, j^{\prime}} x_{i^{\prime} j^{\prime}}^{s^{\prime}} x_{i j}^{s} \otimes m_{i j}^{s} \otimes m_{i^{\prime} j^{\prime}}^{s^{\prime}}
$$

so

$$
\psi_{c}\left(a_{i^{\prime} j^{\prime}}^{s^{\prime}} a_{i j}^{s}\right)=x_{i^{\prime} j^{\prime}}^{s^{\prime}} x_{i j}^{s}=\psi_{c}\left(a_{i^{\prime} j^{\prime}}^{s^{\prime}}\right) \psi_{c}\left(a_{i j}^{s}\right)
$$

for any $s, s^{\prime} \in S$.

Now for $s=1 / 2$ the representation $u^{1 / 2}$ is given by (1.3) so

$$
\left(v^{1 / 2}\right)^{*}=\left(\begin{array}{rr}
\psi_{c}\left(\alpha_{c}\right) & -q \psi_{c}\left(\gamma_{c}^{*}\right) \\
\psi_{c}\left(\gamma_{c}\right) & \psi_{c}\left(\alpha_{c}^{*}\right)
\end{array}\right) \in M_{2}(B(H))
$$

Using the multiplicativity of $\psi_{c}$ and unitarity of $u^{1 / 2}$ we get

$$
\left(\begin{array}{cr}
\psi_{c}\left(\alpha_{c}\right) & -q \psi_{c}\left(\gamma_{c}^{*}\right) \\
\psi_{c}\left(\gamma_{c}\right) & \psi_{c}\left(\alpha_{c}^{*}\right)
\end{array}\right)\left(\begin{array}{rr}
\psi_{c}\left(\alpha_{c}^{*}\right) & \psi_{c}\left(\gamma_{c}^{*}\right) \\
-q \psi_{c}\left(\gamma_{c}\right) & \psi_{c}\left(\alpha_{c}\right)
\end{array}\right)=\left(\begin{array}{cc}
I_{H} & 0 \\
0 & I_{H}
\end{array}\right)
$$


in $M_{2}(B(H))$. Since $v_{d}$ is unitary then $\left(v^{1 / 2}\right)^{*}$ in $(1.34)$ is an unitary matrix in $M_{2}(B(H))$ and from (1.35) we get

$$
\begin{aligned}
\left(\begin{array}{rr}
\psi_{c}\left(\alpha_{c}^{*}\right) & \psi_{c}\left(\gamma_{c}^{*}\right) \\
-q \psi_{c}\left(\gamma_{c}\right) & \psi_{c}\left(\alpha_{c}\right)
\end{array}\right) & =\left(\begin{array}{rr}
\psi_{c}\left(\alpha_{c}\right) & -q \psi_{c}\left(\gamma_{c}^{*}\right) \\
\psi_{c}\left(\gamma_{c}\right) & \psi_{c}\left(\alpha_{c}^{*}\right)
\end{array}\right)^{-1} \\
& =\left(\begin{array}{rr}
\psi_{c}\left(\alpha_{c}\right) & -q \psi_{c}\left(\gamma_{c}^{*}\right) \\
\psi_{c}\left(\gamma_{c}\right) & \psi_{c}\left(\alpha_{c}^{*}\right)
\end{array}\right)^{*}=\left(\begin{array}{cc}
\psi_{c}\left(\alpha_{c}\right)^{*} & \psi_{c}\left(\gamma_{c}{ }^{*}\right. \\
-q \psi_{c}\left(\gamma_{c}^{*}\right)^{*} & \psi_{c}\left(\alpha_{c}^{*}\right)^{*}
\end{array}\right)
\end{aligned}
$$

and $\psi_{c}\left(\alpha_{c}^{*}\right)=\psi_{c}\left(\alpha_{c}\right)^{*}, \psi_{c}\left(\gamma_{c}^{*}\right)=\psi_{c}\left(\gamma_{c}\right)^{*}$. This means that $\psi_{c}: \mathscr{A}_{c} \rightarrow B(H)$ is the unital *-homomorphism. Moreover, $\left(v^{1 / 2}\right)^{*}$ is the unitary matrix of the form $\left(\begin{array}{cc}a & -q c^{*} \\ c & a^{*}\end{array}\right)$ so $a=\psi_{c}\left(\alpha_{c}\right), c=\psi_{c}\left(\gamma_{c}\right)$ has to satisfy the relations (1.2) (cf. [8] remark after (1.33)) and $\psi_{c}$ has the unique extension to a $C^{*}$-representation of $\mathfrak{R}_{c}$ in $B(H)$ [13, Theorem 1.1]. Since $\mathfrak{R}_{c}$ is the unital $C^{*}$-algebra this implies that $\psi_{c} \in \operatorname{Mor}\left(\mathfrak{R}_{c}, C B(H)\right)$ and $v_{d}^{*}=\tau_{d}\left(\mathrm{id}_{d} \otimes \psi_{c}\right) U$. This proves the existence of $\psi_{c}$ satisfying (1.32). The uniqueness of $\psi_{c}$ follows from the fact that any $\psi_{c} \in \operatorname{Mor}\left(\mathfrak{R}_{c}, C B(H)\right)$ is uniquely defined by its values on $\mathscr{A}_{c}$ but if $\psi_{c}$ satisfies (1.32) it has to satisfy (1.33) which completely determines $\psi_{c}$ on $\mathscr{A}_{c}$. Q.E.D.

Remark. Propositions 1.1 and 1.3 show that $G_{c}$ and $G_{d}$ are mutually Pontryagin dual groups. Let $a$ denote one of the letters $c$ or $d$ and $\hat{c}=d, \hat{d}=c$. Then the correspondence $v_{a} \rightarrow \psi_{\hat{a}}$ is natural in the sense that for any unitary representations $v_{a}^{(1)}, v_{a}^{(2)}$ of $G_{a}$ acting on the Hilbert spaces $H_{1}$ and $H_{2}$, respectively and corresponding representations $\psi_{\hat{a}}^{(1)}, \psi_{\hat{a}}^{(2)}$ of $\Re_{\hat{a}}$ we have

$$
\begin{aligned}
& \left\{t \in B\left(H_{1}, H_{2}\right):\left(t \otimes I_{a}\right) v_{a}^{(1)}=v_{a}^{(2)}\left(t \otimes I_{a}\right)\right\} \\
& \quad=\left\{t \in B\left(H_{1}, H_{2}\right): t \psi_{a}^{(1)}(x)=\psi_{a}^{(2)}(x) t \text { for all } x \in \mathfrak{R}_{\hat{a}}\right\}
\end{aligned}
$$

(cf. Remark preceding $\S 4$ in [8]). In particular, unitary representation $v_{a}$ of $G_{a}$ is irreducible if and only if the representation $\psi_{\hat{a}}$ of $\Re_{\hat{a}}$ is irreducible. Let us note also that any operator $t \in B\left(H_{1}, H_{2}\right)$ intertwines representations $v_{c}^{(1)}$ and $v_{c}^{(2)}$ if and only if it maps natural domains into itself $t: D_{1} \rightarrow D_{2}$ and for any $y \in D_{1}$,

$$
\begin{gathered}
t \psi_{d}^{(1)}\left(A_{d}\right) y=\psi_{d}^{(2)}\left(A_{d}\right) t y, \quad t \psi_{d}^{(1)}\left(N_{d}\right) y=\psi_{d}^{(2)}\left(N_{d}\right) t y, \\
t \psi_{d}^{(1)}\left(N_{d}^{*}\right) y=\psi_{d}^{(2)}\left(N_{d}^{*}\right) t y .
\end{gathered}
$$

The last part of this section we devote to the description of unitary representations of quantum Lorentz group in terms of the representations of $G_{c}$ and $G_{d}$. Let $G=(\Re, \Delta)$ be the quantum Lorentz group. The algebra of "continuous functions tending to 0 at infinity" on $G$ is

$$
\mathfrak{R}=\mathfrak{R}_{c} \otimes \mathfrak{R}_{d} .
$$

If

$$
\tilde{\sigma}: \Re_{c} \otimes \Re_{d} \rightarrow \Re_{d} \otimes \Re_{c}
$$

is given by

$$
\tilde{\sigma}(a \otimes x)=U(x \otimes a) U^{*},
$$

where $U$ is the bicharacter (1.11) then

$$
\Delta=\left(\mathrm{id}_{c} \otimes \tilde{\sigma} \otimes \mathrm{id}_{d}\right)\left(\Delta_{c} \otimes \Delta_{d}\right) .
$$


The counit is given by $e=e_{c} \otimes e_{d}$ (cf. (4.9), (4.16), (4.17), and Theorem 4.1 in [8]).

Let $p_{c}=\mathrm{id}_{c} \otimes e_{d}$ and $p_{d}=e_{c} \otimes \mathrm{id}_{d}$ then $p_{c} \in \operatorname{Mor}\left(\Re, \Re_{c}\right), p_{d} \in \operatorname{Mor}\left(\Re, \Re_{d}\right)$. They correspond to the embeddings $G_{c} \rightarrow G, G_{d} \rightarrow G$. The structural theorem for unitary representations of $G$ is related to these embeddings.

Proposition 1.4. i) Let $v \in M(C B(H) \otimes \Re)$ be a unitary representation of $G$ acting on the Hilbert space $H$ and let

$$
v_{c}=\left(\mathrm{id}_{H} \otimes p_{c}\right) v, \quad v_{d}=\left(\mathrm{id}_{H} \otimes p_{d}\right) v .
$$

Then $v_{c} \in M\left(C B(H) \otimes \Re_{c}\right), v_{d} \in M\left(C B(H) \otimes \Re_{d}\right)$ and they are the unitary representations of $G_{c}$ and $G_{d}$, respectively, acting on the Hilbert space $H$ satisfying the compatibility condition

$$
\left(v_{d}\right)_{12}\left(v_{c}\right)_{13}=\left(\operatorname{id}_{H} \otimes \tilde{\sigma}\right)\left(v_{c}\right)_{12}\left(v_{d}\right)_{13}
$$

and

$$
v=\left(v_{c}\right)_{12}\left(v_{d}\right)_{13} .
$$

ii) Let $v_{c} \in M\left(C B(H) \otimes \Re_{c}\right), v_{d} \in M\left(C B(H) \otimes \Re_{d}\right)$ be the unitary representations of $G_{c}$ and $G_{d}$, respectively, acting on the same Hilbert space $H$ and satisfying the compatibility condition (1.38).

Then $v$ defined by (1.39) belongs to $M(C B(H) \otimes \Re)$ and is the unitary representation of $G$.

Proof. The arguments used in the proof of Theorem 4.4 in [8] for the case of finitedimensional representations of $G$ are still valid. One has to check the unitarity condition only but this is obvious because $p_{c}$ and $p_{d}$ are morphisms. Q.E.D.

This proposition reduces the study of unitary representations of $G$ to the study of the pairs of unitary representations of $G_{c}$ and $G_{d}$, respectively, acting in the same Hilbert space and satisfying the compatibility condition (1.38). In view of Propositions 1.1 and 1.3 and Corollary 1.2 we would like to replace the unitary representations $v_{c}, v_{d}$ by corresponding representations $\psi_{d}, \psi_{c}$ of algebras $\mathfrak{R}_{d}$ and $\mathfrak{R}_{c}$, respectively. The main problem is to express the compatibility condition (1.38) in terms of $\psi_{d}$ and $\psi_{c}$. A partial solution to this was given in [8, Proposition 4.5].

Let $\psi_{c} \in \operatorname{Mor}\left(\Re_{c}, C B(H)\right)$ corresponds to $v_{d}$ and let for $a \in \mathfrak{R}_{c}$,

$$
a * \psi_{c}:=\left(\psi_{c} \otimes \mathrm{id}_{c}\right) \Delta_{c}(a), \quad \psi_{c} * a:=\tau_{c}\left(\mathrm{id}_{c} \otimes \psi_{c}\right) \Delta_{c}(a),
$$

where $\tau_{c}$ is a flip $\tau_{c}: \Re_{c} \otimes B(H) \rightarrow B(H) \otimes \Re_{c}$.

It was proved [8, Proposition 4.5] that $v_{c}$ and $v_{d}$ satisfy compatibility condition (1.38) if and only if

$$
\left(a * \psi_{c}\right) v_{c}=v_{c}\left(\psi_{c} * a\right)
$$

for any $a \in\left\{\alpha_{c}, \alpha_{c}^{*}, \gamma_{c}, \gamma_{c}^{*}\right\}$. To abbreviate the notation we shall denote

$$
\alpha=\psi_{c}\left(\alpha_{c}\right), \quad \gamma=\psi_{c}\left(\gamma_{c}\right) .
$$

Then $\alpha \in B(H), \gamma \in B(H)$ and they satisfy relations (1.2). Now (1.40) reads

$$
\begin{gathered}
\left(\alpha \otimes \alpha_{c}-q \gamma^{*} \otimes \gamma_{c}\right) v_{c}=v_{c}\left(\alpha \otimes \alpha_{c}-q \gamma \otimes \gamma_{c}^{*}\right), \\
\left(\alpha^{*} \otimes \alpha_{c}^{*}-q \gamma \otimes \gamma_{c}^{*}\right) v_{c}=v_{c}\left(\alpha^{*} \otimes \alpha_{c}^{*}-q \gamma^{*} \otimes \gamma_{c}\right), \\
\left(\gamma \otimes \alpha_{c}+\alpha^{*} \otimes \gamma_{c}\right) v_{c}=v_{c}\left(\alpha \otimes \gamma_{c}+\gamma \otimes \alpha_{c}^{*}\right), \\
\left(\gamma^{*} \otimes \alpha_{c}^{*}+\alpha \otimes \gamma_{c}^{*}\right) v_{c}=v_{c}\left(\alpha^{*} \otimes \gamma_{c}^{*}+\gamma^{*} \otimes \alpha_{c}\right) .
\end{gathered}
$$


We have

Proposition 1.5. Let $v_{c} \in M\left(C B(H), \mathfrak{R}_{c}\right)$ and $v_{d} \in M\left(C B(H), \mathfrak{R}_{d}\right)$ be the unitary representations of $G_{c}$ and $G_{d}$ acting on the same Hilbert space $H$ and let

$$
D=\sum_{s \in S\left(v_{c}\right)}^{\text {finite }} H^{s}=\sum_{s \in S\left(v_{c}\right)}^{\text {finite }} K^{s} \otimes H_{s}
$$

be the natural domain (1.24) for $v_{c}$. Let $\psi_{d}, \psi_{c}$ be the corresponding representations of algebras $\mathfrak{R}_{d}$ and $\mathfrak{R}_{c}$, respectively on $H$ and

$$
\begin{array}{cc}
A=\psi_{d}\left(A_{d}\right), & N=\psi_{d}\left(N_{d}\right), \\
\alpha=\psi_{c}\left(\alpha_{c}\right), & \gamma=\psi_{c}\left(\gamma_{c}\right)
\end{array}
$$

be the generators of these representations (i.e. A and $N$ have the form (1.25) so they satisfy relations

$$
\left.\begin{array}{l}
A=A^{*}>0, \\
A N=q N A, \\
N^{*}+\frac{1}{1-q^{2}}\left(A^{-2}-A^{2}\right)
\end{array}\right\}
$$

on $D$ and

$$
\left.\begin{array}{c}
\alpha^{*} \alpha+\gamma^{*} \gamma=I_{H}, \\
\alpha \alpha^{*}+q^{2} \gamma^{*} \gamma=I_{H}, \\
\alpha \gamma=q \gamma \alpha, \quad \gamma \gamma^{*}=\gamma^{*} \gamma, \quad \alpha \gamma^{*}=q \gamma^{*} \alpha
\end{array}\right\}
$$

on $H)$.

Then the following conditions are equivalent:

i) $v_{c}$ and $v_{d}$ satisfy the compatibility condition (1.38).

ii) The operator

$$
t=\left(\begin{array}{cc}
\alpha & -q \gamma^{*} \\
\gamma & \alpha^{*}
\end{array}\right) \in M_{2}(B(H))
$$

is an (unitary) intertwining operator for the unitary representations

$$
V_{c}^{(1)}=\left(u^{1 / 2}\right)_{23}\left(v_{c}\right)_{13}, \quad V_{c}^{(2)}=\left(v_{c}\right)_{13}\left(u^{1 / 2}\right)_{23}
$$

acting on $\hat{H}=H \otimes K^{1 / 2}$, i.e.

$$
\left(t \otimes I_{c}\right) V_{c}^{(1)}=V_{c}^{(2)}\left(t \otimes I_{c}\right) .
$$

iii) $a: D \rightarrow D$ for any $a \in\left\{\alpha, \alpha^{*}, \gamma, \gamma^{*}\right\}$ and the following relations

$$
\left.\begin{array}{c}
A \alpha=\alpha A, \quad A \gamma=\frac{1}{q} \gamma A, \\
N \alpha=q \alpha N-q \gamma^{*} A, \quad N \gamma=\gamma N+\frac{1}{q}\left(\alpha^{*} A-\alpha A^{-1}\right), \\
N \alpha^{*}=\frac{1}{q} \alpha^{*} N+\frac{1}{q} \gamma^{*} A^{-1}, \quad N \gamma^{*}=\gamma^{*} N
\end{array}\right\}
$$

are satisfied on D. 
Proof. Let us identify $\hat{H}=H \otimes K^{1 / 2}=H \oplus H$. Then using (1.3) the condition (1.46) means that

$$
\begin{aligned}
& \left(\begin{array}{cc}
v_{c} & 0 \\
0 & v_{c}
\end{array}\right)\left(\begin{array}{cc}
\alpha \otimes \alpha_{c}-q \gamma \otimes \gamma_{c}^{*}, & -q \gamma^{*} \otimes \alpha_{c}-q \alpha^{*} \otimes \gamma_{c}^{*} \\
\alpha \otimes \gamma_{c}+\gamma \otimes \alpha_{c}^{*}, & -q \gamma^{*} \otimes \gamma_{c}+\alpha^{*} \otimes \alpha_{c}^{*}
\end{array}\right) \\
& =\left(\begin{array}{cc}
\alpha \otimes \alpha_{c}-q \gamma^{*} \otimes \gamma_{c}, & -q \gamma^{*} \otimes \alpha_{c}^{*}-q \alpha \otimes \gamma_{c}^{*} \\
\alpha^{*} \otimes \gamma_{c}+\gamma \otimes \alpha_{c}, & -q \gamma \otimes \gamma_{c}^{*}+\alpha^{*} \otimes \alpha_{c}^{*}
\end{array}\right)\left(\begin{array}{cc}
v_{c} & 0 \\
0 & v_{c}
\end{array}\right)
\end{aligned}
$$

and this are exactly the relations (1.41) so i) and ii) are equivalent. Now the intertwiner property (1.46) is equivalent to the statement that $t$ maps the natural domains $\hat{D}_{1}, \hat{D}_{2}$ for $V_{c}^{(1)}$ and $V_{c}^{(2)}$, respectively, into itself and intertwines the generators $\hat{A}_{1}, \hat{N}_{1}$ and $\hat{A}_{2}, \hat{N}_{2}$ of the corresponding representations $\psi_{d}^{(1)}, \psi_{d}^{(2)}$. Since

$$
\hat{D}_{1}=D \otimes K^{1 / 2}=D \oplus D=\hat{D}_{2},
$$

then by (1.45) $a(D) \subset D$ for any $a \in\left\{\alpha, \alpha^{*}, \gamma, \gamma^{*}\right\}$. Moreover, using (1.28) and properties (1.15) and (1.16) of functionals $\varphi_{A}, \varphi_{R}$, and $\varphi_{N}$ we get

$$
\begin{gathered}
\hat{A}_{1}=\left(\mathrm{id}_{\hat{D}_{1}} \otimes \varphi_{R}\right) V_{c}^{(1)}=q^{-1 / 2}\left(\begin{array}{cc}
A & 0 \\
0 & q A
\end{array}\right)=\left(\mathrm{id}_{\hat{D}_{1} \otimes \varphi_{R}}\right) V_{c}^{(2)}=\hat{A}_{2}, \\
\hat{N}_{1}=-q\left(\mathrm{id}_{\hat{D}_{1}} \otimes \varphi_{N}\right) V_{c}^{(1)}=q^{-1 / 2}\left(\begin{array}{cc}
q N & 0 \\
A & N
\end{array}\right), \\
\hat{N}_{2}=-q\left(\mathrm{id}_{\hat{D}_{2}} \otimes \varphi_{N}\right) V_{c}^{(2)}=q^{-1 / 2}\left(\begin{array}{cc}
N & 0 \\
A^{-1} & q N
\end{array}\right) .
\end{gathered}
$$

Now relations $t \hat{A}_{1}=\hat{A}_{2} t, t \hat{N}_{1}=\hat{N}_{2} t$ are the same as relations (1.47) so the equivalence of ii) and iii) follows. Q.E.D.

Let us note that Eqs. (1.43), (1.44), and (1.47) imply that an algebra of continuous functions tending to 0 at infinity on a dual group $\hat{G}$ of the quantum Lorentz group $G$ is "generated" by $\{A, N, \alpha, \gamma\}$ so it contains the algebras $\mathfrak{R}_{c}, \mathfrak{R}_{d}$, but this is not the tensor product of them. Let us denote it symbolically by

$$
\widehat{\mathfrak{R}}=\mathfrak{R}_{c} \odot \Re_{d} .
$$

Then one can define $\hat{\Delta}=\Delta_{c} \odot \Delta_{d}, \hat{e}=e_{c} \odot e_{d}$, and $\hat{\kappa}=\kappa_{c} \odot \kappa_{d}$ which will impose the Hopf $*$-algebra structure on $\hat{\mathfrak{R}}$ or equivalently it will define the Pontryagin dual group $\hat{G}$ on the Hopf $*$-algebra level. Since $G_{c}$ and $G_{d}$ are mutually Pontryagin dual groups one could expect that the quantum Lorentz group is selfdual, but as we see it is not the case. A Hopf algebra structure was recently studied in $[10,6]$.

Proposition 1.5 reduces the problem of classification of the unitary representations of quantum Lorentz group to the problem of classification of four operators $\{A, N, \alpha, \gamma\}$ satisfying on $D$ the relations (1.43), (1.44), and (1.47). For operators $A$ and $N$ it is clear. To incorporate operators $\alpha$ and $\gamma$ into this scheme we use the method of tensor operators.

\section{Tensor Operators}

\subsection{Basic Notions and Operations}

Let $v_{c} \in M\left(C B(H) \otimes \Re_{c}\right)$ be a unitary representation of $G_{c}$ acting on the Hilbert space $H, \psi_{d}$ be the corresponding representation of $\mathfrak{R}_{d}$ and

$$
A=\psi_{d}\left(A_{d}\right), \quad N=\psi_{d}\left(N_{d}\right)
$$


be the generators of this representation. Let

$$
D=\sum_{s \in S\left(v_{c}\right)}^{\mathrm{finite}} H^{s}=\sum_{s \in S\left(v_{c}\right)}^{\mathrm{finite}} K^{s} \otimes H_{s}
$$

be the natural domain for $v_{c}$. We shall consider an *-algebra of linear (in general unbounded) operators (cf. [4])

$$
L^{+}(D)=\left\{T: D_{T}=D, D_{T^{*}} \supset D, T(D) \subset D, T^{*}(D) \subset D\right\} .
$$

Clearly, any $T \in L^{+}(D)$ is a closeable operator on $H$. There is an induced action of $G_{c}$ on the *-algebra $L^{+}(D)$

$$
V_{c}(T):=v_{c}\left(T \otimes I_{c}\right) v_{c}^{*} \text { for any } T \in L^{+}(D)
$$

since it is easy to check that

$$
\left(\mathrm{id}_{L^{+}(D)} \otimes \Delta_{c}\right) V_{c}=\left(V_{c} \otimes \mathrm{id}_{c}\right) V_{c} .
$$

Taking in mind (1.28), (1.15), (1.29), (1.30), and (1.31) we get for generators $\widehat{A}, \hat{N}, \hat{N}^{*}$ of the corresponding representation of $\mathfrak{R}_{d}$,

$$
\begin{aligned}
\hat{A}(T) & =A T A^{-1}, \\
\hat{N}(T) & =N T A^{-1}-q A^{-1} T N, \\
\hat{N}^{\dagger}(T) & =N^{*} T A^{-1}-\frac{1}{q} A^{-1} T N^{*} .
\end{aligned}
$$

In what follows we shall consider $s$-tensor operators, i.e. sets of operators which transforms under the action of $V_{c}$ according to the representation $u^{s}$ of $G_{c}$. Since we will be interested only in the case of $s=0$ and $s=1$ we shall restrict our definition of $s$-tensor operators to this particular case.

Definition 2.1. i) An operator $X \in L^{+}(D)$ is called a scalar operator if

$$
V_{c}(X)=v_{c}\left(X \otimes I_{c}\right) v_{c}^{*}=X \otimes I_{c} .
$$

The set of all scalar operators will be denoted by $\mathscr{T}^{0}(D)$.

ii) A triplet

$$
\mathbf{Y}=\left(\begin{array}{c}
Y_{1} \\
Y_{0} \\
Y_{-1}
\end{array}\right),
$$

where $Y_{1}, Y_{0}, Y_{-1}$ belong to $L^{+}(D)$ is called a vector operator if

$$
V_{c}\left(Y_{j}\right)\left(x \otimes I_{c}\right)=v_{c}\left(Y_{j} \otimes I_{c}\right) v_{c}^{*}\left(x \otimes I_{c}\right)=\sum_{k \in\{-1,0,1\}} Y_{k} x \otimes u_{k j}^{1}
$$

for $j \in\{-1,0,1\}$ and any $x \in D$.

The set of all vector operators will be denoted by $\mathscr{T}^{1}(D)$.

Using (2.4) and (1.20) we see that

1. $X \in \mathscr{T}^{0}(D)$ if and only if

$$
A X=X A, \quad N X=X N, \quad N^{*} X=X N^{*}
$$

on $D$. This implies that $\mathscr{T}^{0}(D)$ is a *algebra. 
2. $\mathbf{Y} \in \mathscr{T}^{1}(D)$ if and only if

$$
\begin{gathered}
A Y_{1}=q Y_{1} A, \quad A Y_{0}=Y_{0} A, \quad A Y_{-1}=\frac{1}{q} Y_{-1} A, \\
N Y_{1}=\frac{1}{q} Y_{1} N, \quad N^{*} Y_{1}=\frac{1}{q} Y_{1} N^{*}+\frac{\sqrt{1+q^{2}}}{q} Y_{0} A, \\
N Y_{0}=Y_{0} N+\frac{\sqrt{1+q^{2}}}{q} Y_{1} A, \quad N^{*} Y_{0}=Y_{0} N^{*}+\frac{\sqrt{1+q^{2}}}{q} Y_{-1} A, \\
N Y_{-1}=q Y_{-1} N+\frac{\sqrt{1+q^{2}}}{q} Y_{0} A, \quad N^{*} Y_{-1}=q Y_{-1} N^{*}
\end{gathered}
$$

on $D$. Clearly, $\mathscr{T}^{1}(D)$ is a vector space and also a bimodule over $\mathscr{T}^{0}(D)$.

For any $\mathbf{Y} \in \mathscr{T}^{1}(D)$ let us define

$$
\mathbf{Y}^{\dagger}=\left(\begin{array}{c}
Y_{1} \\
Y_{0} \\
Y_{-1}
\end{array}\right)^{\dagger}=\left(\begin{array}{c}
-q Y_{-1}^{*} \\
Y_{0}^{*} \\
-\frac{1}{q} Y_{1}^{*}
\end{array}\right)
$$

Then using (2.9) it is easy to show that the map

$$
\mathscr{T}^{1}(D) \ni \mathbf{Y} \mapsto \mathbf{Y}^{\dagger} \in \mathscr{T}^{1}(D)
$$

is an antilinear involution in $\mathscr{T}^{1}(D)$.

Since the tensor product of two $u^{1}$ representations of $G_{c}$ decomposes into a direct sum $u^{0} \oplus u^{1} \oplus u^{2}$ one can expect that there exist two bilinear maps

and

$$
\mathscr{T}^{1}(D) \times \mathscr{T}^{1}(D) \ni(\mathbf{Y}, \mathbf{Z}) \mapsto \mathbf{Y} \bullet \mathbf{Z} \in \mathscr{T}^{0}(D)
$$

$$
\mathscr{T}^{1}(D) \times \mathscr{T}^{1}(D) \ni(\mathbf{Y}, \mathbf{Z}) \mapsto \mathbf{Y} \times \mathbf{Z} \in \mathscr{T}^{1}(D) .
$$

This is really the case and using (2.8), (2.9) one can check that operations

$$
\begin{gathered}
\left(\begin{array}{c}
Y_{1} \\
Y_{0} \\
Y_{-1}
\end{array}\right) \cdot\left(\begin{array}{c}
Z_{1} \\
Z_{0} \\
Z_{-1}
\end{array}\right)=-\frac{1}{q} Y_{-1} Z_{1}+Y_{0} Z_{0}-q Y_{1} Z_{-1}, \\
\left(\begin{array}{c}
Y_{1} \\
Y_{0} \\
Y_{-1}
\end{array}\right) \times\left(\begin{array}{c}
Z_{1} \\
Z_{0} \\
Z_{-1}
\end{array}\right)=\frac{1}{\sqrt{1+q^{2}}}\left(\begin{array}{c}
Y_{0} Z_{1}-q^{2} Y_{1} Z_{0} \\
q\left(Y_{-1} Z_{1}-Y_{1} Z_{-1}\right)+\left(1-q^{2}\right) Y_{0} Z_{0} \\
Y_{-1} Z_{0}-q^{2} Y_{0} Z_{-1}
\end{array}\right)
\end{gathered}
$$

called the scalar product and the vector product of vector operators satisfy requirements (2.11) and (2.12), respectively.

\subsection{Basic Maps}

Tensor operators on $D$ are intertwiners for the appropriate actions of $G_{c}$. We use this fact to describe a structure of such operators and related operations described above. 
Let $X \in \mathscr{T}^{0}(D)$ be a scalar operator on the natural domain $D$ for the unitary representation $v_{c}$ of $G_{c}$ [i.e. $D$ has the form (2.1)]. Then by (2.5) it is an intertwining operator for $v_{c}$ on $D$. Since $X$ is closeable and $v_{c}$ restricted to $H^{s}$ is $u^{s} \otimes I_{H_{s}}$ and $u^{s}$ is an irreducible representation of $G_{c}$ then

$$
X=\sum_{s \in S\left(v_{c}\right)} I_{K^{s}} \otimes \Phi_{s}^{0}(X),
$$

where $\Phi_{s}^{0}(X) \in B\left(H_{s}\right)$ is a bounded operator on $H_{s}$ for any $s \in S\left(v_{c}\right)$. In what follows we shall identify $K^{s}$ for $s \geqq 1$ as the $q$-symmetric part of $\bigotimes_{n=1}^{2 s} K^{1 / 2}$ where $K^{1 / 2}$ is a 2-dimensional carrier Hilbert space of the fundamental representation $u^{1 / 2}$ [cf. (1.3)]. For $s=1$ we shall abbreviate $K^{1}=K$. Let $\left\{f_{m}^{s}: m=-s,-s+1, \ldots, s-1, s\right\}$ be the canonical basis (1.19) in $K^{s}$. For $\mathbf{Y} \in \mathscr{T}^{1}(D)$ let us define a map

$$
\begin{gathered}
\Phi(\mathbf{Y}): K \otimes D \rightarrow D, \\
\Phi(\mathbf{Y})\left(f_{j}^{1} \otimes x\right)=Y_{j} x .
\end{gathered}
$$

It is clear using (2.7) that $\Phi(\mathbf{Y})$ is an intertwining operator for the $G_{c}$ actions by $u^{1} \otimes v_{c}$ on $K \otimes D$ and $v_{c}$ on $D$. Since for any $s \in S$ the tensor product of representations $u^{1}$ and $u^{s}$ has a decomposition into a direct sum $\bigoplus_{s^{\prime}=|s-1|}^{s+1} u^{s^{\prime}}$ we define a proximity relations for spins $s, s^{\prime} \in S$. We shall say that $s^{\prime}$ is near $s$ and denote it by $s^{\prime} \sim s$ whenever $u^{s^{\prime}}$ is contained in the tensor product of $u^{1}$ and $u^{s}$. This means that

$$
s^{\prime} \sim s \text { if and only if } \begin{cases}s^{\prime} \in\{s-1, s, s+1\} & \text { for } s \geqq 1, \\ s^{\prime} \in\{1 / 2,3 / 2\} & \text { for } s=1 / 2, \\ s^{\prime}=1 & \text { for } s=0 .\end{cases}
$$

To describe in more detail the structure of operator $\Phi(\mathbf{Y})$ and operations mentioned previously we shall fix some intertwiners for $G_{c}$ actions. To this end we use technique of diagrams.

By one vertical line we denote an element of $K^{1 / 2}$. Since $u^{1 / 2} \otimes u^{1 / 2}=u^{0} \oplus u^{1}$ and the corresponding decomposition is

$$
K^{1 / 2} \otimes K^{1 / 2}=K^{0} \oplus K^{1}
$$

(this corresponds to the decomposition into $q$-antisymmetric and $q$-symmetric tensors) then there are two intertwiners:

$$
E: K^{0}=C \rightarrow K^{1 / 2} \otimes K^{1 / 2}, \quad E^{\prime}: K^{1 / 2} \otimes K^{1 / 2} \rightarrow C .
$$

We shall denote them by

$$
E=\bigwedge, \quad E^{\prime}=\bigvee
$$

and fix as

$$
E(1)=f_{-1 / 2}^{1 / 2} \otimes f_{1 / 2}^{1 / 2}-q f_{1 / 2}^{1 / 2} \otimes f_{-1 / 2}^{1 / 2},
$$

and

$$
\begin{gathered}
E^{\prime}\left(f_{-1 / 2}^{1 / 2} \otimes f_{-1 / 2}^{1 / 2}\right)=0=E^{\prime}\left(f_{1 / 2}^{1 / 2} \otimes f_{1 / 2}^{1 / 2}\right), \\
E^{\prime}\left(f_{-1 / 2}^{1 / 2} \otimes f_{1 / 2}^{1 / 2}\right)=-\frac{1}{q}, \quad E^{\prime}\left(f_{1 / 2}^{1 / 2} \otimes f_{-1 / 2}^{1 / 2}\right)=1 .
\end{gathered}
$$


One can check that

$$
\aleph^{=}=\bigvee
$$

and

$$
E^{\prime} \cdot E=\bigcap=-\frac{1+q^{2}}{q} .
$$

Let us define an intertwiner

$$
\sigma: K^{1 / 2} \otimes K^{1 / 2} \rightarrow K^{1 / 2} \otimes K^{1 / 2}
$$

by

$$
\sigma=\searrow=q^{\frac{1}{2}}||+q^{-\frac{1}{2}} \bigwedge .
$$

Then $\sigma$ satisfies a quadratic equation

$$
\sigma^{2}+\left(q^{-3 / 2}-q^{1 / 2}\right) \sigma-\frac{1}{q}=0
$$

and $\sigma$ has two eigenvalues: $q^{1 / 2}$ on $K^{1}=K$ and $-q^{-3 / 2}$ on $K^{0}=C$. One can also check that $\sigma$ satisfies the braid equation. Using (2.24) and (2.22) we have

$$
X=1 M \quad X Y=V \mid
$$

Let $x \in \bigotimes_{n=1}^{2 s} K^{1 / 2}$ then $x \in K^{s}$ if and only if

$$
\overbrace{\|\| \ldots \| \ldots .|W|}^{2 s \text { ines }}=0,
$$

where $E^{\prime}$ is applied to any pair $(i, i+1)$ of lines.

We shall also need a symmetrization operator (intertwiners)

$$
\hat{\Psi}_{\left(s+\frac{1}{2}\right) s}: K^{1 / 2} \otimes K^{s} \rightarrow K^{s+\frac{1}{2}}, \quad \Psi_{(s+1) s}: K \otimes K^{s} \rightarrow K^{s+1} .
$$

Since $K^{s+\frac{1}{2}}$ and $K^{s+1}$ can be identified as subspaces of $K^{1 / 2} \otimes K^{s}$ and $K \otimes K^{s}$, respectively, we can take

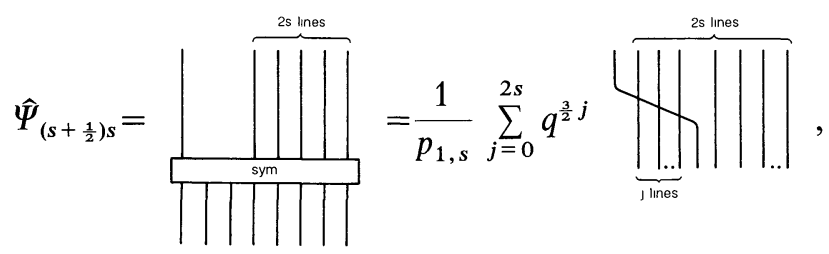


where $p_{1, s}=\sum_{j=0}^{2 s} q^{2 j}$ is a normalization factor,

$$
\Psi_{(s+1) s}=\overbrace{\prod_{\|\| \|}^{2 s} \mid \|}^{2 s \text { ines }}=\frac{1}{p_{2, s}} \sum_{\substack{j, k=0 \\ j+k \leqq 2 s}} q^{\frac{3}{2}(2 j+k)}
$$

and $p_{2, s}=\sum_{j, k=0, j+k \leqq 2 s} q^{(2 j+k)}$ is a normalization factor. They are projections as can be seen using (2.26) and (2.24). In particular,

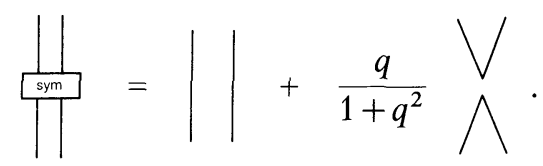

Now we fix intertwiners

$$
\Psi_{s^{\prime} s}: K \otimes K^{s} \rightarrow K^{s^{\prime}}
$$

for $s^{\prime} \sim s$ :
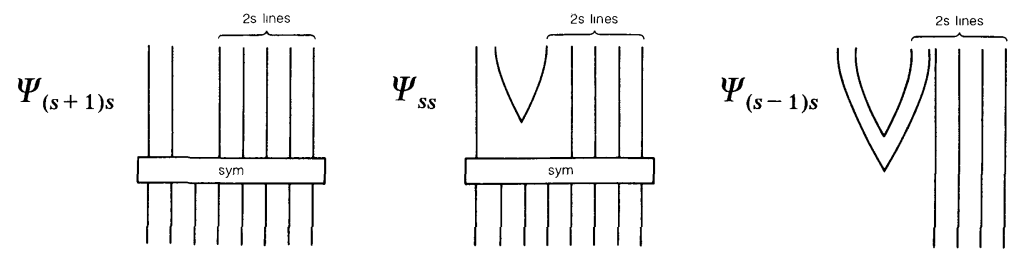

and let

$$
\Theta: C \rightarrow K \otimes K, \quad \Theta^{*}: K \otimes K \rightarrow C, \quad T: K \rightarrow K \otimes K
$$

be fixed operators given by
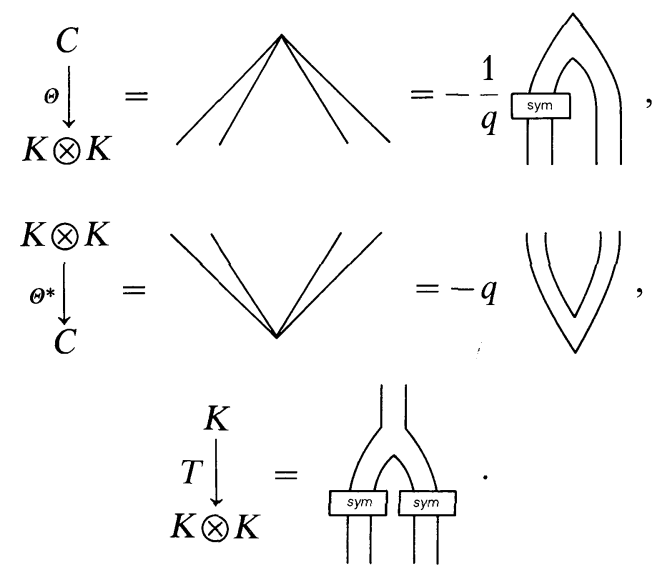

Now for $\mathbf{Y} \in \mathscr{T}^{1}(D)$

$$
\Phi(\mathbf{Y}): K \otimes D=\sum_{s \in S\left(v_{c}\right)} K \otimes K^{s} \otimes H_{s} \rightarrow \sum_{s^{\prime} \in S\left(v_{c}\right)} K^{s^{\prime}} \otimes H_{s^{\prime}}
$$


can be written as

$$
\Phi(\mathbf{Y})=\sum_{\substack{s^{\prime}, s_{s} \in\left(v_{c}\right) \\ s^{\prime} \sim s}} \Psi_{s^{\prime} s} \otimes \Phi_{s^{\prime} s}^{1}(\mathbf{Y}),
$$

where $\Phi_{s^{\prime} s}^{1}(\mathbf{Y}) \in B\left(H_{s}, H_{s^{\prime}}\right)$ for $s, s^{\prime} \in S\left(v_{c}\right), s^{\prime} \sim s$ and completely determine $\Phi(\mathbf{Y})$ :

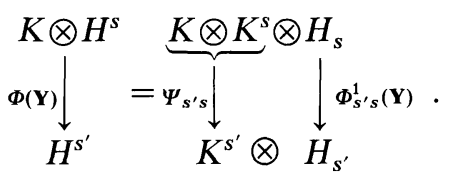

Since

$$
\Theta(1)=-\frac{1}{q} f_{-1}^{1} \otimes f_{1}^{1}+f_{0}^{1} \otimes f_{0}^{1}-q f_{1}^{1} \otimes f_{-1}^{1}
$$

and

$$
\begin{aligned}
T f_{1}^{1} & =\frac{1}{\sqrt{1+q^{2}}}\left(f_{0}^{1} \otimes f_{1}^{1}-q^{2} f_{1}^{1} \otimes f_{0}^{1}\right), \\
T f_{0}^{1} & =\frac{1}{\sqrt{1+q^{2}}}\left[q\left(f_{-1}^{1} \otimes f_{1}^{1}-f_{1}^{1} \otimes f_{-1}^{1}\right)+\left(1-q^{2}\right) f_{0}^{1} \otimes f_{0}^{1}\right], \\
T f_{-1}^{1} & =\frac{1}{\sqrt{1+q^{2}}}\left(f_{-1}^{1} \otimes f_{0}^{1}-q^{2} f_{0}^{1} \otimes f_{-1}^{1}\right)
\end{aligned}
$$

we get by (2.16) for $\mathbf{Y}, \mathbf{Z} \in \mathscr{T}^{1}(D)$

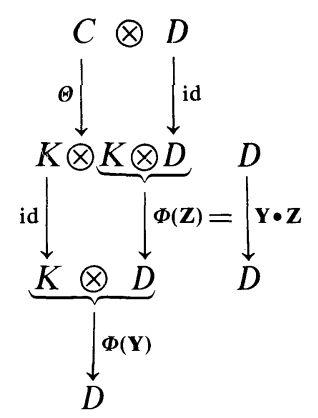

and

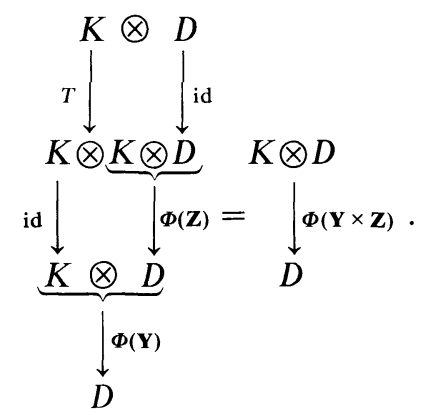


Moreover, since for $x \in D$

$$
\Phi(\mathbf{Y})^{*} x=\sum_{j \in\{-1,0,1\}} f_{j}^{1} \otimes Y_{j}^{*} x
$$

and

$$
\begin{gathered}
\Theta^{*}\left(f_{1}^{1} \otimes f_{j}^{1}\right)=-q \delta_{j,-1} \\
\Theta^{*}\left(f_{0}^{1} \otimes f_{j}^{1}\right)=\delta_{j, 0}, \\
\Theta^{*}\left(f_{-1}^{1} \otimes f_{j}^{1}\right)=-\frac{1}{q} \delta_{j,-1}
\end{gathered}
$$

we have

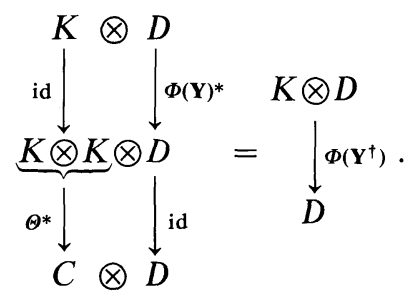

Now we can compute $\Phi_{s}^{0}(\mathbf{Y} \bullet \mathbf{Z}), \Phi_{s^{\prime} s}^{1}(\mathbf{Y} \times \mathbf{Z})$, and $\Phi_{s^{\prime} s}^{1}\left(\mathbf{Y}^{\dagger}\right)$ in terms of $\Phi_{s^{\prime} s}^{1}(\mathbf{Y})$ and $\Phi_{s^{\prime} s}^{1}(\mathbf{Z})$.

a) The Scalar Product of Vector Operators. From Eqs. (2.15), (2.16), (2.13) using (2.38) and (2.36) we have

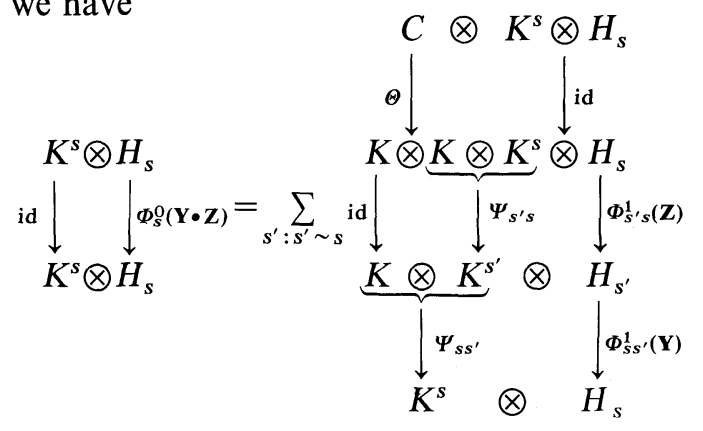

so

$$
\Phi_{s}^{0}(\mathbf{Y} \bullet \mathbf{Z})=\sum_{s^{\prime}: s^{\prime} \sim s} \lambda_{s s^{\prime} s} \Phi_{s s^{\prime}}^{1}(\mathbf{Y}) \Phi_{s^{\prime} s}^{1}(\mathbf{Z})
$$

where

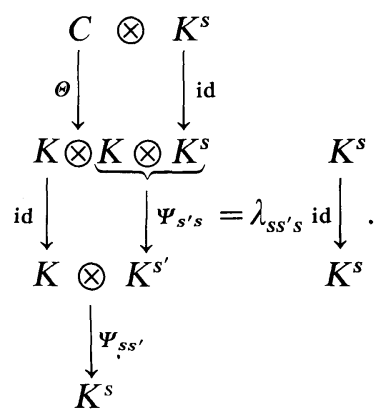


The $\lambda_{s s^{\prime} s}$ for $s^{\prime} \sim s$ in (2.42) can be computed and

$$
\begin{aligned}
& \lambda_{s(s+1) s}=-\frac{[2 s+3]_{q}}{q^{3}[2 s+1]_{q}}, \\
& \lambda_{s s s}=\frac{[2 s+2]_{q}}{q^{2}\left(1+q^{2}\right)[2 s]_{q}} \text { for } s>0, \\
& \lambda_{s(s-1) s}=-\frac{1}{q} \quad \text { for } s \geqq 1 .
\end{aligned}
$$

For more details see Appendix.

b) The Vector Product of Vector Operators. We compute $\Phi_{s^{\prime} s}^{1}(\mathbf{Y} \times \mathbf{Z})$. Using definition (2.14) and Eqs. (2.39), (2.37), (2.36) we have

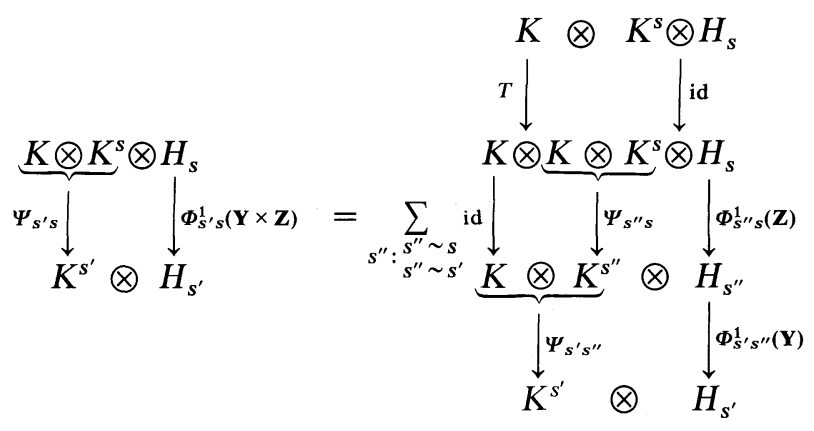

and

$$
\Phi_{s^{\prime} s}^{1}(\mathbf{Y} \times \mathbf{Z})=\sum_{\substack{s^{\prime \prime} \\ s^{\prime \prime} \sim s \\ s^{\prime \prime} \sim s^{\prime}}} \varrho_{s^{\prime} s^{\prime \prime} s} \Phi_{s^{\prime} s^{\prime \prime}}^{1}(\mathbf{Y}) \Phi_{s^{\prime \prime} s}^{1}(\mathbf{Z})
$$

where

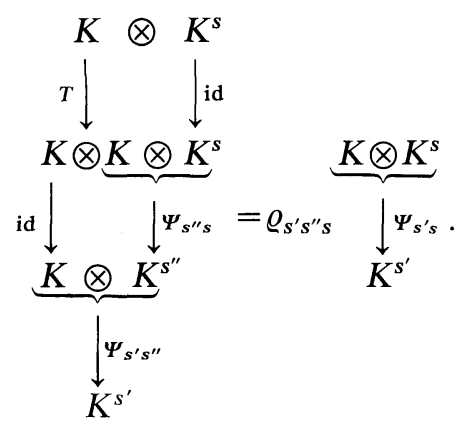

One can compute $\varrho_{s^{\prime} s^{\prime \prime} s}$ for $s^{\prime} \sim s^{\prime \prime}, s^{\prime \prime} \sim s, s^{\prime} \sim s$ (see Appendix):

$$
\begin{gathered}
\varrho_{(s-1)(s-1) s}=\frac{q}{1+q^{2}} \text { for } s \geqq 1, \quad \varrho_{s(s-1) s}=1 \quad \text { for } s \geqq 1, \\
\varrho_{s(s+1) s}=-\frac{[2 s]_{q}[2 s+3]_{q}}{[2 s+1]_{q}[2 s+2]_{q}} \text { for } s \geqq 0, \quad \varrho_{(s+1)(s+1) s}=-\frac{[2 s+4]_{q}}{q\left(1+q^{2}\right)[2 s+2]_{q}}, \\
\varrho_{(s-1) s s}=-\frac{[2 s+2]_{q}}{q\left(1+q^{2}[2 s]_{q}\right.} \text { for } s \geqq 1, \quad \varrho_{s s s}=-\frac{1+q^{(4 s+2)}}{q\left(1+q^{2}\right)[2 s]_{q}} \text { for } s>0 \\
\varrho_{(s+1) s s}=\frac{q}{1+q^{2}} .
\end{gathered}
$$


c) The Adjoint of Vector Operator. From (2.40), (2.10) and since

$$
\Phi(\mathbf{Y})^{*}=\sum_{s^{\prime}, s: s^{\prime} \sim s}\left(\Psi_{s s^{\prime}}\right)^{*} \otimes \Phi_{s^{\prime} s}^{1}(\mathbf{Y})^{*}
$$

we get

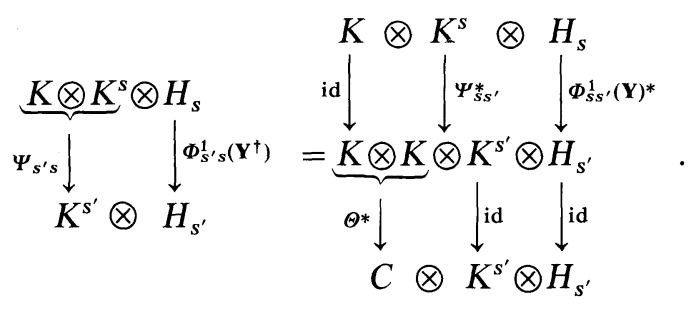

The computation of

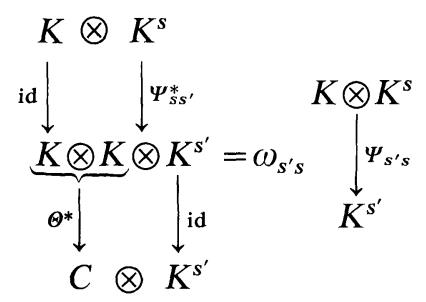

gives (cf. Appendix):

$$
\omega_{(s+1) s}=-\frac{1}{q}, \quad \omega_{s s}=1, \quad \omega_{(s-1) s}=-q .
$$

Comparing this with (2.47) one obtains:

$$
\begin{aligned}
\Phi_{(s+1) s}^{1}\left(\mathbf{Y}^{\dagger}\right) & =-\frac{1}{q} \Phi_{s(s+1)}^{1}(\mathbf{Y})^{*}, \\
\Phi_{s s}^{1}\left(\mathbf{Y}^{\dagger}\right) & =\Phi_{s s}^{1}(\mathbf{Y})^{*}, \\
\Phi_{(s-1) s}^{1}\left(\mathbf{Y}^{\dagger}\right) & =-q \Phi_{s(s-1)}^{1}(\mathbf{Y})^{*} .
\end{aligned}
$$

At the end let us remark that since $\mathscr{T}^{1}(D)$ is a bimodule over $\mathscr{T}^{0}(D)$ we have for $\mathbf{Y} \in \mathscr{T}^{1}(D)$ and $X \in \mathscr{T}^{0}(D)$ that $\mathbf{Y} \cdot X \in \mathscr{T}^{1}(D)$ and $X \cdot \mathbf{Y} \in \mathscr{T}^{-1}(D)$,

$$
\begin{aligned}
& \Phi_{s^{\prime} s}^{1}(\mathbf{Y} \cdot X)=\Phi_{s^{\prime} s}^{1}(\mathbf{Y}) \Phi_{s}^{0}(X), \\
& \Phi_{s^{\prime} s}^{1}(X \cdot \mathbf{Y})=\Phi_{s^{\prime}}^{0}(X) \Phi_{s^{\prime} s}^{1}(\mathbf{Y}) .
\end{aligned}
$$

\section{Irreducible Unitary Representations}

This section is devoted mainly to the proof of Theorem 0.1. We describe all irreducible unitary representations of quantum Lorentz group, i.e. irreducible families of four operators $\{\alpha, \gamma, A, N\}$ satisfying relations (1.43), (1.44), and (1.47). Let us define

$$
C=\frac{1}{1+q^{2}}\left[\left(1-q^{2}\right)^{2} N^{*} N+q^{2} A^{2}+A^{-2}\right]
$$




$$
\begin{gathered}
\mathbf{Z}=\left(\begin{array}{c}
Z_{1} \\
Z_{0} \\
Z_{-1}
\end{array}\right)=\left(\begin{array}{c}
\frac{q\left(1-q^{2}\right)}{\sqrt{1+q^{2}}} N A \\
-\frac{1}{1+q^{2}}\left[\left(1-q^{2}\right)^{2} N^{*} N+A^{-2}-A^{2}\right] \\
-\frac{1-q^{2}}{q \sqrt{1+q^{2}}} N^{*} A
\end{array}\right), \\
X=\frac{1}{\sqrt{1+q^{2}}}\left[q\left(1-q^{2}\right) \gamma N-\left(q^{2} \alpha^{*} A+\alpha A^{-1}\right)\right]
\end{gathered}
$$

and

$$
\mathbf{Y}=\left(\begin{array}{c}
Y_{1} \\
Y_{0} \\
Y_{-1}
\end{array}\right)=\left(\begin{array}{c}
\left(1-q^{2}\right) \alpha^{*} N+\gamma^{*} A^{-1} \\
\frac{1}{\sqrt{1+q^{2}}}\left[q\left(1-q^{2}\right) \gamma N+\left(\alpha^{*} A-\alpha A^{-1}\right)\right] \\
\gamma A
\end{array}\right)
$$

Using (1.43),(1.44), and (1.47) one can check that operators $C, X$ satisfy (2.8) and Z, Y satisfy (2.9) so

$$
C \in \mathscr{T}^{0}(D), \quad X \in \mathscr{T}^{0}(D), \quad \mathbf{Z} \in \mathscr{T}^{1}(D), \quad \mathbf{Y} \in \mathscr{T}^{1}(D) .
$$

The operator $C$ is essentially selfadjoint on $D$ and commutes with $A, N$, and $N^{*}$ so it is a Casimir operator for $S_{q} U(2)$. Since the action of operators $A$ and $N$ on $D$ is known, then using (1.25), (1.26), and (1.20) one can compute [cf. (2.15)] that

$$
\begin{aligned}
\Phi_{s}^{0}(C) & =C_{s} I_{H_{s}} & \text { for } \quad s \in S\left(v_{c}\right), \\
C_{s} & =\frac{q^{-2 s}\left(1+q^{4 s+2}\right)}{1+q^{2}} & \text { for } \quad s \in S .
\end{aligned}
$$

Taking into account that $H^{s}$ is invariant space for $A, N$, and $N^{*}$ we see that $\Phi_{s^{\prime} s}^{1}(\mathbf{Z})$ can be $\neq 0$ only if $s^{\prime}=s$ then by (2.30) and (2.34) we have

$$
\begin{gathered}
\Phi_{s^{\prime} s}^{1}(\mathbf{Z})=\delta_{s^{\prime}, s^{2}} \mathscr{Z}_{s} I_{H_{s}} \quad \text { for } \quad s \in S\left(v_{c}\right), \\
\mathscr{Z}_{s}=\frac{q\left(q^{-2 s}-q^{2 s}\right)}{\sqrt{1+q^{2}}} \text { for } \quad s \in S,
\end{gathered}
$$

so $\Phi(\mathbf{Z})=\sum_{s \in S\left(v_{c}\right)} \Psi_{s s} \otimes \mathscr{Z}_{s} I_{H_{s}}$.

Using again commutation relations (1.43), (1.44), and (1.47) one checks that $X$ commutes with $A, N, \alpha, \gamma$, and their adjoints so it is a Casimir operator for $S_{q} L(2, C)$. Moreover, $A, N, \alpha, \gamma$ and their adjoints are bounded on $H^{s}$ for any $s \in S\left(v_{c}\right)$ and $H^{s}$ is invariant subspace for $X$ so $X$ is a normal operator and $D$ is an essential domain for it. Now relations (1.43), (1.44), (1.47) imply

$$
\begin{gathered}
X^{*}=X \cdot C+\mathbf{Y} \cdot \mathbf{Z}, \\
\mathbf{Y}^{\dagger}=\sqrt{1+q^{2}} \mathbf{Y} \times \mathbf{Z}-q^{2} \mathbf{Y} \cdot C, \\
\mathbf{Y} \cdot \mathbf{Y}=X^{2}-\left(1+q^{2}\right) I,
\end{gathered}
$$




$$
\mathbf{Y} \times \mathbf{Y}=\frac{1-q^{2}}{\sqrt{1+q^{2}}} X \cdot \mathbf{Y} .
$$

This shows that the set of operators $\{A, N, \alpha, \gamma\}$ satisfying (1.43), (1.44), and (1.47) can be replaced by the set $\left\{A, N, X, Y_{1}, Y_{0}, Y_{-1}\right\}$ of operators satisfying (2.9) and (3.7)-(3.10). For irreducible set of operators we have an additional condition that

$$
X=\mathscr{X}_{0} I_{D} \text { for some complex number } \mathscr{X}_{0} \in C \text {. }
$$

Remark. Let us note that the $C^{*}$-algebra associated with quantum spheres of Podleś [7] can be generated equivalently by coordinates of vector operator $\mathbf{Y}$ such that

$$
\mathbf{Y}^{\dagger}=\mathbf{Y}, \quad \mathbf{Y} \cdot \mathbf{Y}=\varrho I, \quad \mathbf{Y} \times \mathbf{Y}=\lambda \mathbf{Y}
$$

for some real $\varrho$ and $\lambda$. We see that in our case the $C^{*}$-algebra generated by coordinates of $\mathbf{Y}$ and $\mathbf{Y}^{\dagger}$ in the case of an irreducible unitary representation of $G$ will correspond to "complexification" of quantum sphere.

Let $p$ denote a minimal spin in the unitary representation $v_{c}$, i.e.

$$
p=\min \left\{s: s \in S\left(v_{c}\right)\right\},
$$

then Eqs. (3.7) and (3.8) by using (2.50), (2.41), (2.44), and (3.5), (3.6) can be expressed in terms of the corresponding mappings and we get for $s \in S\left(v_{c}\right)$ :

$$
\begin{aligned}
\mathscr{X}_{0}^{*} I_{H_{s}} & =\mathscr{X}_{0} C_{s} I_{H_{s}}+\lambda_{s s s} \mathscr{Z}_{s} \Phi_{s s}^{1}(\mathbf{Y}), \\
-\frac{1}{q} \Phi_{s(s+1)}^{1}(\mathbf{Y})^{*} & =\left[\sqrt{1+q^{2}} \varrho_{(s+1) s s} \mathscr{Z}_{s}-q^{2} C_{2}\right] \Phi_{(s+1) s}^{1}(\mathbf{Y}), \\
\Phi_{s s}^{1}(\mathbf{Y})^{*}= & {\left[\sqrt{1+q^{2}} \varrho_{s s s} \mathscr{Z}_{s}-q^{2} C_{s}\right] \Phi_{s s}^{1}(\mathbf{Y})-\mathscr{X}_{0} \mathscr{Z}_{s} I_{H_{s}}, } \\
-q \Phi_{s(s-1)}^{1}(\mathbf{Y})^{*} & =\left[\sqrt{1+q^{2}} \varrho_{(s-1) s s} \mathscr{Z}_{s}-q^{2} C_{s}\right] \Phi_{(s-1) s}^{1}(\mathbf{Y}) .
\end{aligned}
$$

Let us note that $\Phi_{(s-1) s}^{1}(\mathbf{Y})=0$ whenever $s<p+1$. Analogously, Eqs. (3.9) and (3.10) lead to

$$
\begin{aligned}
& \lambda_{s(s+1) s} \Phi_{s(s+1)}^{1}(\mathbf{Y}) \Phi_{(s+1) s}^{1}(\mathbf{Y})+\lambda_{s s s} \Phi_{s s}^{1}(\mathbf{Y})^{2}+\lambda_{s(s-1) s} \Phi_{s(s-1)}^{1}(\mathbf{Y}) \Phi_{(s-1) s}^{1}(\mathbf{Y}) \\
& \quad=\left[\mathscr{X}_{0}^{2}-\left(1+q^{2}\right)\right] I_{H_{s}}, \\
& \varrho_{s(s+1) s} \Phi_{s(s+1)}^{1}(\mathbf{Y}) \Phi_{(s+1) s}^{1}(\mathbf{Y})+\varrho_{s s s} \Phi_{s s}^{1}(\mathbf{Y})^{2}+\varrho_{s(s-1) s} \Phi_{s(s-1)}^{1}(\mathbf{Y}) \Phi_{(s-1) s}^{1}(\mathbf{Y}) \\
& \quad=\frac{1-q^{2}}{\sqrt{1+q^{2}}} \mathscr{X}_{0} \Phi_{s s}^{1}(\mathbf{Y}), \\
& \varrho_{(s+1)(s+1) s} \Phi_{(s+1)(s+1)}^{1}(\mathbf{Y}) \Phi_{(s+1) s}^{1}(\mathbf{Y})+\varrho_{(s+1) s s} \Phi_{(s+1) s}^{1}(\mathbf{Y}) \Phi_{s s}^{1}(\mathbf{Y}) \\
& \quad=\frac{1-q^{2}}{\sqrt{1+q^{2}}} \mathscr{X}_{0} \Phi_{(s+1) s}^{1}(\mathbf{Y}), \\
& \varrho_{(s-1)(s-1) s} \Phi_{(s-1)(s-1)}^{1}(\mathbf{Y}) \Phi_{(s-1) s}^{1}(\mathbf{Y})+\varrho_{(s-1) s s} \Phi_{(s-1) s}^{1}(\mathbf{Y}) \Phi_{s s}^{1}(\mathbf{Y}) \\
& \quad=\frac{1-q^{2}}{\sqrt{1+q^{2}}} \mathscr{X}_{0} \Phi_{(s-1) s}^{1}(\mathbf{Y}) .
\end{aligned}
$$

At first let us observe that for an irreducible representation $v$ by (3.14)-(3.16) the support (1.23) of corresponding $v_{c}$ is contained in the set $S_{p}$ [cf. (0.2)]. 
Moreover, since $\lambda_{\text {sss }} \mathscr{Z}_{s} \neq 0$ for $s>0$ by (2.43) and (3.6) then (3.13) determines $\Phi_{s s}^{1}(\mathbf{Y})$. For $s=0$ (it can occur only if $p=0$ ) there is no $\lambda_{000}$ [the map $\Phi_{00}^{1}(\mathbf{Y})$ does not exist since 0 is not near 0$]$ and (3.13) is an additional condition for $\mathscr{X}_{0}$. Since by (3.5) $C_{0}=1$ we see that if $p=0$ then $\mathscr{X}_{0}=\overline{\mathscr{X}}_{0}$, i.e. $\mathscr{X}_{0}$ has to be real. In what follows we shall consider separately two cases: $p>0$ and $p=0$. Since the decomposition of $v_{c}$ into direct sum of irreducible representations is unique it is clear that the minimal spin $p$ is invariant of unitary equivalence. We shall denote also $S(v)=S\left(v_{c}\right)$ and call it the support of $v$.

Proposition 3.1. i) Let $v$ be an unitary irreducible representation of quantum Lorentz group $G$ with minimal spin $p>0$ and let $\mathscr{X}_{0}$ be the value of Casimir operator $X$ for $v$. Then $\mathscr{X}_{0} \in \mathscr{E}_{p}$.

ii) Let $\mathscr{X}_{0} \in \mathscr{E}_{p}$ for some $p \in S$ and $p>0$.

Then there exists a unique (up to unitary equivalence) irreducible unitary representation $v$ of $G$ with minimal spin $p$ and for which the value of Casimir operator $X$ is $\mathscr{X}_{0}$.

Moreover $S(v)=S_{p}$ and for any $s \in S(v) \operatorname{dim} H_{s}=1$.

Proof. From (3.13) we get for $s \geqq p$

$$
\Phi_{s s}^{1}(\mathbf{Y})=\mathscr{Y}_{s s} I_{H_{s}} \quad \text { with } \quad \mathscr{Y}_{s s}=\frac{\overline{\mathscr{X}}_{0}-C_{s} \mathscr{X}_{0}}{\lambda_{s s s} \mathscr{Z}_{s}} .
$$

Now for $s=p$ from (3.17) and (3.18) we get the minimal equations

$$
\begin{aligned}
& \lambda_{p(p+1) p} \Phi_{p(p+1)}^{1}(\mathbf{Y}) \Phi_{(p+1) p}^{1}(\mathbf{Y})+\lambda_{p p p} \Phi_{p p}^{1}(\mathbf{Y})^{2}=\left[\mathscr{X}_{0}^{2}-\left(1+q^{2}\right)\right] I_{H_{p}}, \\
& \varrho_{p(p+1) p} \Phi_{p(p+1)}^{1}(\mathbf{Y}) \Phi_{(p+1) p}^{1}(\mathbf{Y})+\varrho_{p p p} \Phi_{p p}^{1}(\mathbf{Y})^{2}=\frac{1-q^{2}}{\sqrt{1+q^{2}}} \mathscr{X}_{0} \Phi_{p p}^{1}(\mathbf{Y}) .
\end{aligned}
$$

Eliminating $\Phi_{p(p+1)}^{1}(\mathbf{Y}) \Phi_{(p+1) p}^{1}(\mathbf{Y})$ from (3.22), (3.23) and substituting $\mathscr{Y}_{p p}$ from (3.21) and using (3.6) and (2.43) we get the additional condition on $\mathscr{X}_{0}$ :

$$
q^{-2 p}\left(1+q^{4 p}\right)\left|\mathscr{X}_{0}\right|^{2}-\left(\mathscr{X}_{0}^{2}+\overline{\mathscr{X}}_{0}^{2}\right)=\frac{q^{2-4 p}\left(1-q^{4 p}\right)^{2}}{1+q^{2}}
$$

which implies that $\mathscr{X}_{0} \in \mathscr{E}_{p}$ and this proves i).

Now let $\mathscr{X}_{0} \in \mathscr{E}_{p}$ for some $p>0$, i.e. [cf. (0.3)]

$$
\mathscr{X}_{0}=\frac{q}{\sqrt{1+q^{2}}}\left[\left(q^{p}+q^{-p}\right) \cos \varphi+i\left(q^{-p}-q^{p}\right) \sin \varphi\right]
$$

for some $\varphi \in[0,2 \pi[$. To prove the existence of $v$ one has to show that Eqs. (3.13)-(3.20) have an unique solution under the condition that $v$ has to be irreducible. From (3.22) using (3.24), (3.6), and (2.43) we get

$$
\mathscr{Y}_{s s}=-q\left[\frac{q^{-s}-q^{s}}{q^{-(s+1)}+q^{s+1}}\left(q^{p}+q^{-p}\right) \cos \varphi+i \frac{q^{-s}+q^{s}}{q^{-(s+1)}-q^{s+1}}\left(q^{-p}-q^{p}\right) \sin \varphi\right] .
$$

Then Eq. (3.15) is fulfilled identically. From (3.14) we get

$$
\Phi_{s(s+1)}^{1}(\mathbf{Y})^{*}=q^{2 s+3} \Phi_{(s+1) s}^{1}(\mathbf{Y})
$$

and (3.16) imply that for $s>p$,

$$
q^{2 s+1} \Phi_{s(s-1)}^{1}(\mathbf{Y})^{*}=\Phi_{(s-1) s}^{1}(\mathbf{Y})
$$


which is equivalent (3.26) by passing to adjoint and replacing $s \rightarrow s+1$. Taking this into account let for $s \geqq p$,

$$
R_{s}^{+}=\Phi_{s(s+1)}^{1}(\mathbf{Y}) \Phi_{(s+1) s}^{1}(\mathbf{Y})=q^{2 s+3}\left|\Phi_{(s+1) s}^{1}(\mathbf{Y})\right|^{2}
$$

and for $s>p$

$$
R_{s}^{-}=\Phi_{s(s-1)}^{1}(\mathbf{Y}) \Phi_{(s-1) s}^{1}(\mathbf{Y})=q^{-(2 s+1)}\left|\Phi_{(s-1) s}^{1}(\mathbf{Y})\right|^{2},
$$

then $R_{s}^{+} \geqq 0$ and $R_{s}^{-} \geqq 0$. On the other hand since $\Phi_{s s}^{1}(\mathbf{Y})$ is known then (3.17), (3.18) is the set of linear equations for $R_{s}^{+}, R_{s}^{-}$and we get

$$
\begin{aligned}
\left|\Phi_{(s+1) s}^{1}(\mathbf{Y})\right|^{2}= & q^{2} \frac{[s+p+1]_{q}[s-p+1]_{q}}{[2 s+2]_{q}[2 s+3]_{q}} \\
& \times\left[\left(q^{s+1}+q^{-(s+1)}\right)^{2}-4 \cos ^{2} \varphi\right] I_{H_{s}} . \\
\left|\Phi_{(s-1) s}^{1}(\mathbf{Y})\right|^{2}=q^{4 s+4} & \frac{[s+p]_{q}[s-p]_{q}}{[2 s]_{q}[2 s+1]_{q}}\left[\left(q^{s}+q^{-s)}\right)^{2}-4 \cos ^{2} \varphi\right] I_{H_{s}} .
\end{aligned}
$$

The minimal value of $\left(q^{s}+q^{-s}\right)$ is 2 for $s=0$ so $\left|\Phi_{(s+1) s}^{1}(\mathbf{Y})\right|^{2}>0$ for $s \geqq p$ and $\left|\Phi_{(s-1) s}^{1}(\mathbf{Y})\right|^{2}>0$ for $s>p$. We denote

$$
\begin{aligned}
& \mathscr{Y}_{(s+1) s}=q \sqrt{\frac{[s+p+1]_{q}[s-p+1]_{q}}{[2 s+2]_{q}[2 s+3]_{q}}\left[\left(q^{s+1}+q^{-(s+1)}\right)^{2}-4 \cos ^{2} \varphi\right]}, \\
& \mathscr{Y}_{(s-1) s}=q^{2 s+2} \sqrt{\frac{[s+p]_{q}[s-p]_{q}}{[2 s]_{q}[2 s+1]_{q}}\left[\left(q^{s}+q^{-s}\right)^{2}-4 \cos ^{2} \varphi\right] .}
\end{aligned}
$$

Let now $e_{p} \neq 0$ be a normalized vector in $H_{p}$. Then $e_{p}$ is an eigenvector for $\Phi_{p p}^{1}(\mathbf{Y})$ with the eigenvalue $\mathscr{Y}_{p p}$. Using (3.30) we see that $\Phi_{(p+1) p}^{1}(\mathbf{Y}) e_{p} \neq 0$ and normalizing it we get the unit vector $e_{p+1}$ such that

$$
\Phi_{(p+1) p}^{1}(\mathbf{Y}) e_{p}=\mathscr{Y}_{(p+1) p} e_{p+1} .
$$

Now Eq. (3.19) shows that $e_{p+1}$ is an eigenvector for $\Phi_{(p+1)(p+1)}^{1}(\mathbf{Y})$ with the eigenvalue $\mathscr{Y}_{(p+1)(p+1)}$. Moreover by (3.28)

$$
\Phi_{p(p+1)}^{1}(\mathbf{Y}) e_{p+1}=q^{2 p+3} \mathscr{Y}_{(p+1) p} e_{p} .
$$

In the same manner we can define $e_{p+2}$ and by induction we see that starting from a unit vector $e_{p} \in H_{p}$ we get for $s \in S_{p}$ a unit vector $e_{s} \in H_{s}$ such that

$$
\begin{gathered}
\Phi_{s s}^{1}(\mathbf{Y}) e_{s}=\mathscr{Y}_{s s} e_{s}, \\
\Phi_{(s+1) s}^{1}(\mathbf{Y}) e_{s}=\mathscr{Y}_{(s+1) s} e_{s+1}, \quad \Phi_{(s-1) s}^{1}(\mathbf{Y}) e_{s}=\mathscr{Y}_{(s-1) s} e_{s-1} .
\end{gathered}
$$

Let $H_{s}^{\prime}$ be a vector space spaned by $e_{s^{\text {. }}}$ Clearly $\operatorname{dim} H_{s}^{\prime}=1$ and

$$
D^{\prime}=\sum_{s \in S_{p}} K^{s} \otimes H_{s}^{\prime} \subset D
$$

is invariant under the action of $A, N, N^{*}, Y_{j}, Y_{j}^{*}(j=-1,0,1)$ so by irreducibility $D^{\prime}=D$ since $D^{\prime}$ is the natural domain for the action of $v$ on the invariant subspace $H=\sum_{s \in S_{p}}^{\oplus} K^{s} \otimes H_{s}^{\prime}$ and $H_{s}^{\prime}=H_{s}$ for all $s \in S_{p}$. This ends the proof of ii). Q.E.D. 
For minimal spin $p=0$ we have

Proposition 3.2. i) Let $v$ be an unitary irreducible representation of quantum Lorentz group $G$ with minimal spin $p=0$ and let $\mathscr{X}_{0}$ be the value of Casimir operator $X$ for $v$. Then $\mathscr{X}_{0} \in \mathscr{E}_{0}$.

ii) Let $\mathscr{X}_{0} \in \mathscr{E}_{0}$.

Then there exists a unique (up to unitary equivalence) irreducible unitary representation $v$ of $G$ with minimal spin $p=0$ and for which the value of Casimir operator $X$ is $\mathscr{X}_{0}$.

Moreover

1. If $\mathscr{X}_{0}= \pm \sqrt{1+q^{2}}$ then $v$ is a 1-dimensional representation.

2. If $\left.\mathscr{X}_{0} \in\right]-\sqrt{1+q^{2}}, \sqrt{1+q^{2}}\left[\right.$ then $S(v)=S_{0}$ and for any $s \in S(v) \operatorname{dim} H_{s}=1$.

Proof. If $v$ is an irreducible unitary representation of $G$ with minimal spin $p=0$ then we know that $\mathscr{X}_{0}$ is real. Using the fact that $[\mathrm{cf} .(2.43)] \lambda_{010}=-\frac{[3]_{q}}{\mathrm{q}^{3}}$ Eq. (3.17) gives

$$
\Phi_{01}^{1}(\mathbf{Y}) \Phi_{10}^{1}(\mathbf{Y})=\frac{q^{3}}{[3]_{q}}\left[\left(1+q^{2}\right)-\mathscr{X}_{0}^{2}\right] \text {. }
$$

Now from (3.14) and (3.5), (3.6) for $s=0$ we get as before [cf. (3.26)] that $\Phi_{01}^{1}(\mathbf{Y})=q^{3} \Phi_{10}^{1}(\mathbf{Y})$ and

$$
\left|\Phi_{10}^{1}(\mathbf{Y})\right|^{2}=\frac{1}{q^{3}}\left[\left(1+q^{2}\right)-\mathscr{X}_{0}^{2}\right] I_{H_{0}}
$$

has to be $\geqq 0$ so $\mathscr{X}_{0} \in \mathscr{E}_{0}$. This proves i).

Let $\mathscr{X}_{0} \in \mathscr{E}_{0}$. Then we can write

$$
\mathscr{X}_{0}=\sqrt{1+q^{2}} \cos \varphi \text { for some } \varphi \in[0,2 \pi[.
$$

Let us assume that $\mathscr{X}_{0}= \pm \sqrt{1+q^{2}}$. Then by (3.34), (3.35) $\Phi_{10}^{1}(\mathbf{Y})=0$ and $\Phi_{01}^{1}(\mathbf{Y})=0$ so $H^{0}=K^{0} \otimes H_{0}=H_{0}$ is invariant under the action of $Y_{j}, Y_{j}^{*}(j=-1,0,1)$ and $Y_{j}=0$ on $H^{0}$. Since by (1.25), (1.26), (1.20) $A=1, N=0=N^{*}$ on $H^{0}$ and by (3.4) $Y_{-1}=\gamma A=\gamma=0$ we have by (1.44) that $\alpha= \pm 1$. From (3.3) we get

$$
\begin{array}{lll}
\{A, N, \alpha, \gamma\}=\{1,0,1,0\} \quad \text { for } \quad \mathscr{X}_{0}=-\sqrt{1+q^{2}}, \\
\{A, N, \alpha, \gamma\}=\{1,0,-1,0\} \quad \text { for } \quad \mathscr{X}_{0}=\sqrt{1+q^{2}} .
\end{array}
$$

Since in any case $\{A, N, \alpha, \gamma\}$ is the set of commuting operators it is clear that $\operatorname{dim} H^{0}=1$.

We assume now that $\left|\mathscr{X}_{0}\right|<\sqrt{1+q^{2}}$. Then we can argue as in the case $p>0$. For $s>0$ we get by (3.13) and (3.36),

$$
\Phi_{s s}^{1}(\mathbf{Y})=\mathscr{Y}_{s s} I_{H_{s}} \quad \text { with } \quad \mathscr{Y}_{s s}=-\left(1+q^{2}\right) \frac{q^{-s}-q^{s}}{q^{-(s+1)}+q^{s+1}} \cos \varphi .
$$

Using this we get for $s \in S(v)$ :

$$
\begin{aligned}
\left|\Phi_{(s+1) s}^{1}(\mathbf{Y})\right|^{2}= & q^{2} \frac{[s+1]_{q}^{2}}{[2 s+2]_{q}[2 s+3]_{q}}\left[\left(q^{s+1}+q^{-(s+1)}\right)^{2}\right. \\
& \left.-\left(q+q^{-1}\right)^{2} \cos ^{2} \varphi\right] I_{H_{s}} \\
\left|\Phi_{(s-1) s}^{1}(\mathbf{Y})\right|^{2}= & q^{4 s+4} \frac{[s]_{q}^{2}}{[2 s]_{q}[2 s+1]_{q}}\left[\left(q^{s}+q^{-s}\right)^{2}\right. \\
& \left.-\left(q+q^{-1}\right)^{2} \cos ^{2} \varphi\right] I_{H_{s}} .
\end{aligned}
$$


Since in this case $\cos ^{2} \varphi<1$ we see that $\Phi_{(s+1) s}^{1}(\mathbf{Y}) \neq 0$ for $s \in S_{0}$ and for $s>0$ also $\Phi_{(s-1) s}^{1}(\mathbf{Y}) \neq 0$. In the same manner as before we can construct a set of normalized vectors $\left\{e_{s}: e_{s} \in H_{s}\right.$ for $\left.s \in S_{0}\right\}$ such that

$$
\begin{gathered}
\Phi_{s s}^{1}(\mathbf{Y}) e_{s}=\mathscr{Y}_{s s} e_{s}, \\
\Phi_{(s+1) s}^{1}(\mathbf{Y}) e_{s}=\mathscr{Y}_{(s+1) s} e_{s+1}, \quad \Phi_{(s-1) s}^{1}(\mathbf{Y}) e_{s}=\mathscr{Y}_{(s-1) s} e_{s-1},
\end{gathered}
$$

where $\mathscr{Y}_{s s}$ is given by (3.37) and

$$
\begin{aligned}
& \mathscr{Y}_{(s+1) s}=q \sqrt{\frac{[s+1]_{q}^{2}}{[2 s+2]_{q}[2 s+3]_{q}}\left[\left(q^{s+1}+q^{-(s+1)}\right)^{2}-\left(q+q^{-1}\right)^{2} \cos ^{2} \varphi\right]}, \\
& \mathscr{Y}_{(s-1) s}=q^{2 s+2} \sqrt{\frac{[s]_{q}^{2}}{[2 s]_{q}[2 s+1]_{q}}\left[\left(q^{s}+q^{-s}\right)^{2}-\left(q+q^{-1}\right)^{2} \cos ^{2} \varphi\right]},
\end{aligned}
$$

and we conclude that $S(v)=S_{0}$ and $\operatorname{dim} H_{s}=1$ for any $s \in S_{0}$. This ends the proof of ii). Q.E.D.

The proof of Theorem 0.1 is now a straightforward corollary.

Remark. Let $v$ be an unitary representation of $G$ and $X$ be the corresponding Casimir operator. Then $X$ is normal operator and it is clear that $S p X$ is an invariant of unitary equivalence. Let $\Sigma_{q}=\bigcup_{p \in S} \mathscr{E}_{p}$ then above propositions show that $\operatorname{SpX} \subset \Sigma_{q}$. Moreover $\operatorname{SpX}$ and multiplicities does not completely determine the representation since for $\mathscr{X}_{0}= \pm \sqrt{1+q^{2}}$ and $\mathscr{X}_{0}= \pm \frac{1}{\sqrt{1+q^{2}}}\left(q^{3 / 2}+q^{1 / 2}\right)$ there are two types of nonequivalent irreducible unitary representations: the minimal spin $p \in\{0,1\}$ or $p \in\{0,1 \mid 2\}$ respectively. It is also clear that the minimal spin $p$ is also invariant of unitary equivalence so for irreducible representations it is not a function of $\mathscr{X}_{0}$.

The last part of this section we devote to the description of the irreducible unitary representations of quantum Lorentz group in terms of operators $\{A, N, \alpha, \gamma\}$. Let us note that if $v$ is an irreducible unitary representation of the quantum Lorentz group $G$ with minimal spin $p \in S$ acting on the Hilbert space $H=\sum_{s \in S(v)}^{\oplus} H^{s}$ then for any $s \in S(v)=S_{p}$ the space $H^{s}$ is canonically isomorphic to $K^{s}$ (remember that $\left.\operatorname{dim} H_{s}=1\right)$ :

$$
K^{s} \ni x \mapsto x \otimes e_{s} \in K^{s} \otimes H_{s}=H^{s} .
$$

We shall identify elements of $H^{s}$ with elements of $K^{s}$ by this isomorphism. Using this we have

Theorem 3.3. i) Let $v$ be an unitary irreducible representation of the quantum Lorentz group $G$ with minimal spin $p \in S$ and Casimir operator value $\mathscr{X}_{0} \in \mathscr{E}_{p}$ (i.e.

$$
\mathscr{X}_{0}=\left\{\begin{array}{lll}
\frac{q}{\sqrt{1+q^{2}}}\left[\left(q^{p}+q^{-p}\right) \cos \varphi+i\left(q^{-p}-q^{p}\right) \sin \varphi\right] & \text { for } & p>0 \\
\sqrt{1+q^{2}} \cos \varphi & \text { for } & p=0
\end{array}\right.
$$

for some $\varphi \in\left[0,2 \pi\left[\right.\right.$ ) acting on the Hilbert space $H=\cdot \cdot \sum_{s \in S(v)}^{\oplus} H^{s}$.

Then there exists for any $s \in S_{p}$ an orthonormal basis

$$
\left\{f_{m}^{s}: m=-s,-s+1, \ldots, s-1, s\right\}
$$


(cf. (1.19)) in $H^{s}$ such that

$$
\begin{aligned}
& A f_{m}^{s}=q^{m} f_{m}^{s}, \\
& N f_{m}^{s}=q^{-s} \sqrt{[s-m]_{q}[s+m+1]_{q}} f_{m+1}^{s}, \\
& N^{*} f_{m}^{s}=q^{-s} \sqrt{[s+m]_{q}[s-m+1]_{q}} f_{m-1}^{s}, \\
\alpha f_{m}^{s}= & q^{-s} \sqrt{[s-m]_{q}[s+m]_{q}} a_{s-1} f_{m}^{s-1} \\
& -\left[\frac{q^{m}+q^{-m}}{1-q^{2}} q^{s+1} b_{s}+i \frac{q^{-m}-q^{m}}{1-q^{2}} q^{s+1} c_{s}\right] f_{m}^{s} \\
& -q^{s+1} \sqrt{[s-m+1]_{q}[s+m+1]_{q}} a_{s} f_{m}^{s+1}, \\
\alpha^{*} f_{m}^{s}= & -q^{s} \sqrt{[s-m]_{q}[s+m]_{q}} a_{s-1} f_{m}^{s-1} \\
& -\left[\frac{q^{m}+q^{-m}}{1-q^{2}} q^{s+1} b_{s}-i \frac{q^{-m}-q^{m}}{1-q^{2}} q^{s+1} c_{s}\right] f_{m}^{s} \\
+ & q^{-s-1} \sqrt{[s-m+1]_{q}[s+m+1]_{q}} a_{s} f_{m}^{s+1}, \\
\gamma f_{m}^{s}= & q^{-m} \sqrt{[s+m]_{q}[s+m-1]_{q}} a_{s-1} f_{m-1}^{s-1} \\
+ & \sqrt{[s+m]_{q}[s-m+1]_{q}}\left(b_{s}+i c_{s}\right) f_{m}^{s-1} \\
+ & q^{m-1} \sqrt{[s-m+1]_{q}[s-m+2]_{q}} a_{s} f_{m-1}^{s+1}, \\
\gamma^{*} f_{m}^{s}= & q^{m} \sqrt{[s-m]_{q}[s-m-1]_{q}} a_{s-1} f_{m+1}^{s-1} \\
+ & \sqrt{[s-m]_{q}[s+m+1]_{q}}\left(b_{s}-i c_{s}\right) f_{m+1}^{s} \\
+ & q^{-m-1} \sqrt{[s+m+1]_{q}[s+m+2]_{q}} a_{s} f_{m+1}^{s+1},
\end{aligned}
$$

where

$$
a_{s}=\left\{\begin{array}{l}
\frac{q^{2(s+1)}}{[2 s+2]_{q}} \sqrt{\frac{[s+p+1]_{q}[s-p+1]_{q}}{[2 s+1]_{q}[2 s+3]_{q}}\left[\left(q^{s+1}+q^{-s-1}\right)^{2}-4 \cos ^{2} \varphi\right]} \\
\frac{q^{2(s+1)}}{[2 s+2]_{q}} \sqrt{\frac{[s+1]_{q}^{2}}{[2 s+1]_{q}[2 s+3]_{q}}\left[\left(q^{s+1}+q^{-s-1}\right)^{2}-\left(q+q^{-1}\right)^{2} \cos ^{2} \varphi\right]}
\end{array}\right.
$$

$b_{s}+i c_{s}=\left\{\begin{array}{lll}\frac{\left(1-q^{2}\right) q^{s}}{\left(1+q^{2 s}\right)\left(1+q^{2(s+1)}\right)}\left(q^{p}+q^{-p}\right) \cos \varphi & \\ \quad+i \frac{\left(1-q^{2}\right) q^{s}}{\left(1-q^{2 s}\right)\left(1-q^{2(s+1)}\right)}\left(q^{-p}-q^{p}\right) \sin \varphi & \text { for } & p>0 \\ \frac{\left(1-q^{2}\right) q^{s}}{\left(1+q^{2 s}\right)\left(1+q^{2(s+1)}\right)}\left(q+q^{-1}\right) \cos \varphi & \text { for } & p=0 .\end{array}\right.$

ii) Let $p \in S$ and $\varphi \in[0,2 \pi[$ be fixed. Then operators $\{A, N, \alpha, \gamma\}$ defined by (3.44)-(3.48) satisfy the commutation relations (1.43),(1.44), (1.47) on $D=\sum_{s \in S_{p}} K^{s}$ and 
they describe an irreducible unitary representation $v$ of $G$ with minimal spin $p$ and Casimir operator value (3.43).

Proof. Let $\left\{f_{m}^{s}: m=-s,-s+1, \ldots, s\right\}$ be the orthonormal basis (1.19) in $K^{s} \sim H^{s}$ then (3.44) is clear by (1.20) and (1.25), (1.26). To compute (3.46) and (3.47) let us remark that from (3.4) and (3.3) we get

$$
\gamma=Y_{-1} A^{-1}, \quad \alpha^{*}=\frac{1}{\sqrt{1+q^{2}}}\left(Y_{0}-X\right) A^{-1}
$$

so

$$
\begin{gathered}
\gamma f_{m}^{s}=q^{-m} Y_{-1} f_{m}^{s}, \\
\alpha^{*} f_{m}^{s}=\frac{q^{-m}}{\sqrt{1+q^{2}}}\left(Y_{0} f_{m}^{s}-\mathscr{X}_{0} f_{m}^{s}\right),
\end{gathered}
$$

and it is enough to compute $Y_{-1} f_{m}^{s}$ and $Y_{0} f_{m}^{s}$. By the definitions (2.16) and (2.34) we know that for $j \in\{-1,0,1\}$,

$$
\begin{aligned}
Y_{j} f_{m}^{s}= & \Phi(\mathbf{Y})\left(f_{j}^{1} \otimes f_{m}^{s}\right)=\Psi_{(s-1) s}\left(f_{j}^{1} \otimes f_{m}^{s}\right) \mathscr{Y}_{(s-1) s}+\Psi_{s s}\left(f_{j}^{1} \otimes f_{m}^{s}\right) \mathscr{Y}_{s s} \\
& +\Psi_{(s+1) s}\left(f_{j}^{1} \otimes f_{m}^{s}\right) \mathscr{Y}_{(s+1) s}
\end{aligned}
$$

and for $j=-1,0$ we have to compute $\Psi_{s^{\prime} s}\left(f_{j}^{1} \otimes f_{m}^{s}\right)$ for $s^{\prime} \sim s$. At first using (2.30), (2.28), (2.27), and (2.21) we can compute

$$
\begin{aligned}
\Psi_{(s-1) s}\left(f_{-1}^{1} \otimes f_{s}^{s}\right) & =\frac{1}{q^{2}} f_{s-1}^{s-1}, \\
\Psi_{s s}\left(f_{-1}^{1} \otimes f_{s}^{s}\right) & =-\frac{q^{2 s-2}}{\sqrt{[2 s]_{q}}} f_{s-1}^{s}, \\
\Psi_{(s+1) s}\left(f_{-1}^{1} \otimes f_{s}^{s}\right) & =\frac{q^{4 s} \sqrt{1+q^{2}}}{\sqrt{[2 s+1]_{q}[2 s+2]_{q}}} f_{s-1}^{s+1} .
\end{aligned}
$$

Now we can use the fact that $\Psi_{s^{\prime} s}$ is an intertwining operator for $u^{1} \otimes u^{s}$ and $u^{s^{\prime}}$,

$$
u^{s^{\prime}}\left(\Psi_{s^{\prime} s} \otimes I_{c}\right)=\left(\Psi_{s^{\prime} s} \otimes I_{c}\right)\left(u^{1} \otimes u^{s}\right) .
$$

Applying $\varphi_{N^{*}}$ to both sides of (3.56) and using (1.29), (1.30), (1.28) we get

$$
N_{s^{\prime}}^{*} \Psi_{s^{\prime} s}=\Psi_{s^{\prime} s}\left(N_{1}^{*} \otimes A_{s}+A_{1}^{-1} \otimes N_{s}^{*}\right)
$$

and since $N_{1}^{*} f_{-1}^{1}=0$ then we get a recurrence formula

$$
N_{s^{\prime}}^{*} \Psi_{s^{\prime} s}\left(f_{-1}^{1} \otimes f_{m}^{s}\right)=q \Psi_{s^{\prime} s}\left(f_{-1}^{1} \otimes N_{s}^{*} f_{m}^{s}\right) .
$$

Starting with $m=s$ by (3.55) and (1.20) we obtain from (3.57):

$$
\begin{aligned}
& \Psi_{(s-1) s}\left(f_{-1}^{1} \otimes f_{m}^{s}\right)=\frac{1}{q^{2}} \sqrt{\frac{[s+m-1]_{q}[s+m]_{q}}{[2 s-1]_{q}[2 s]_{q}}} f_{m-1}^{s-1}, \\
& \Psi_{s s}\left(f_{-1}^{1} \otimes f_{m}^{s}\right)=-q^{s+m-2} \frac{\sqrt{[s+m]_{q}[s-m+1]_{q}}}{[2 s]_{q}} f_{m-1}^{s}, \\
& \Psi_{(s+1) s}\left(f_{-1}^{1} \otimes f_{m}^{s}\right)=q^{2(s+m)} \sqrt{\frac{[s-m+1]_{q}[s-m+2]_{q}}{[2 s+1]_{q}[2 s+2]_{q}}} f_{m-1}^{s+1} \text {. }
\end{aligned}
$$


Combining this with (3.54), (3.52) and using (3.25), (3.32), (3.33) or (3.38), (3.41), (3.42) we arrive at (3.47).

Now we are ready to compute also $\Psi_{s^{\prime} s}\left(f_{0}^{1} \otimes f_{m}^{s}\right)$. Applying $\varphi_{N}$ to both sides of (3.56) and using (1.28), (1.15) we get

$$
N_{s^{\prime}} \Psi_{s^{\prime} s}=\Psi_{s^{\prime} s}\left(N_{1} \otimes A_{s}+A_{1}^{-1} \otimes N_{s}\right) .
$$

By (1.20) and (3.58) this gives

$$
\begin{aligned}
\Psi_{(s-1) s}\left(f_{0}^{1} \otimes f_{m}^{s}\right) & =-\sqrt{1+q^{2}} q^{s+m-2} \sqrt{\frac{[s+m]_{q}[s-m]_{q}}{[2 s-1]_{q}[2 s]_{q}}} f_{m}^{s-1}, \\
\Psi_{s s}\left(f_{0}^{1} \otimes f_{m}^{s}\right) & =\frac{1-q^{2(s+m)}\left(1+q^{2}\right)+q^{4 s+2}}{q\left(1-q^{4 s}\right)} \sqrt{1+q^{2}} f_{m}^{s}, \\
\Psi_{(s+1) s}\left(f_{0}^{1} \otimes f_{m}^{s}\right) & =\sqrt{1+q^{2}} q^{s+m} \sqrt{\frac{[s-m+1]_{q}[s+m+1]_{q}}{[2 s+1]_{q}[2 s+2]_{q}}} f_{m}^{s+1} .
\end{aligned}
$$

This by (3.54), (3.53) and (3.32), (3.33), (3.25) or (3.38), (3.41), (3.42) leads to (3.46). Taking adjoints in (3.47) and (3.46) we get (3.48) and (3.45). This proves i). One can check by computations that the operators $\{A, N, \alpha, \gamma\}$ defined by (3.44)-(3.48) satisfy the commutation relations (1.43), (1.44), (1.47) on $D=\sum_{s \in S_{p}} K^{s}$. It is clear that the minimal spin is $p$ and using (3.3) we get (3.43). Using Proposition 1.5 and Remark after the proof of Proposition 1.3 we end the proof of ii). Q.E.D.

\section{A. Appendix}

In this section we give examples of calculations of coefficients listed in Sect. 2.

a) The Scalar Product of Vector Operators. By using the properties of maps previously defined we compute for example $\lambda_{s(s+1) s}$ and this means that we have to compute diagram (2.42) for $s^{\prime}=s+1$. Using the definition (2.27) and the fact that $p_{1, s}=[2 s+1]_{q}$ we shall compute at first a simpler diagram

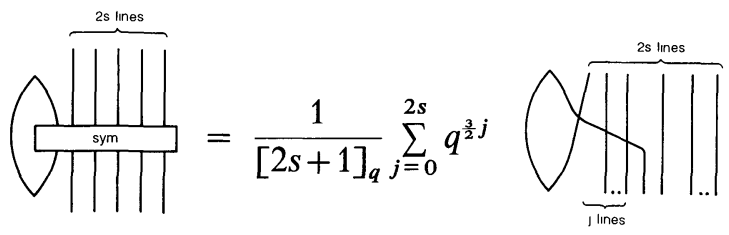

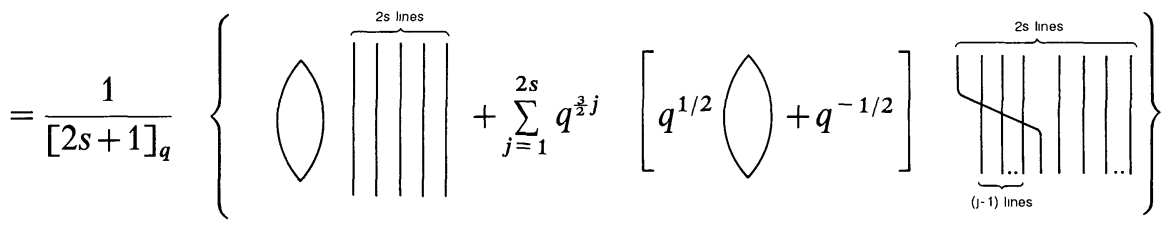

$$
\begin{aligned}
& =-\frac{1}{[2 s+1]_{q}}\left(\frac{1}{q}+q+q \sum_{j=0}^{2 s-1} q^{2 j}\right) \overbrace{\|\|}^{2 s \text { ines }}=Q_{2 s} \overbrace{\|\|}^{2 s \text { ines }}
\end{aligned}
$$


where

$$
Q_{2 s}=-\frac{[2 s+2]_{q}}{q[2 s+1]_{q}}
$$

In the second row we have used (2.27), (2.23) and since $\sigma$ has the eigenvalue $q^{1 / 2}$ on symmetric tensors we got the last equality.

Using this we have from (2.30) and (2.31)

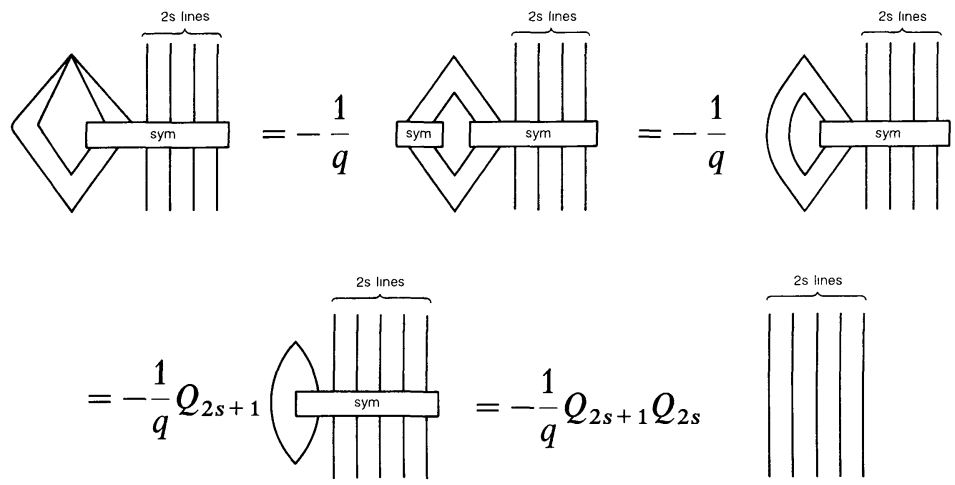

where we used (2.29) to omit the first symmetrization and

$$
\lambda_{s(s+1) s}=-\frac{1}{q} Q_{2 s+1} Q_{2 s}=-\frac{[2 s+3]_{q}}{q^{3}[2 s+1]_{q}} .
$$

The rest values can be obtained in the same manner.

b) The Vector Product of Vector Operators. We compute diagram (2.45) in the case $\varrho_{\text {sss. }}$

Using (2.29), property (2.22) and again (2.29) we get
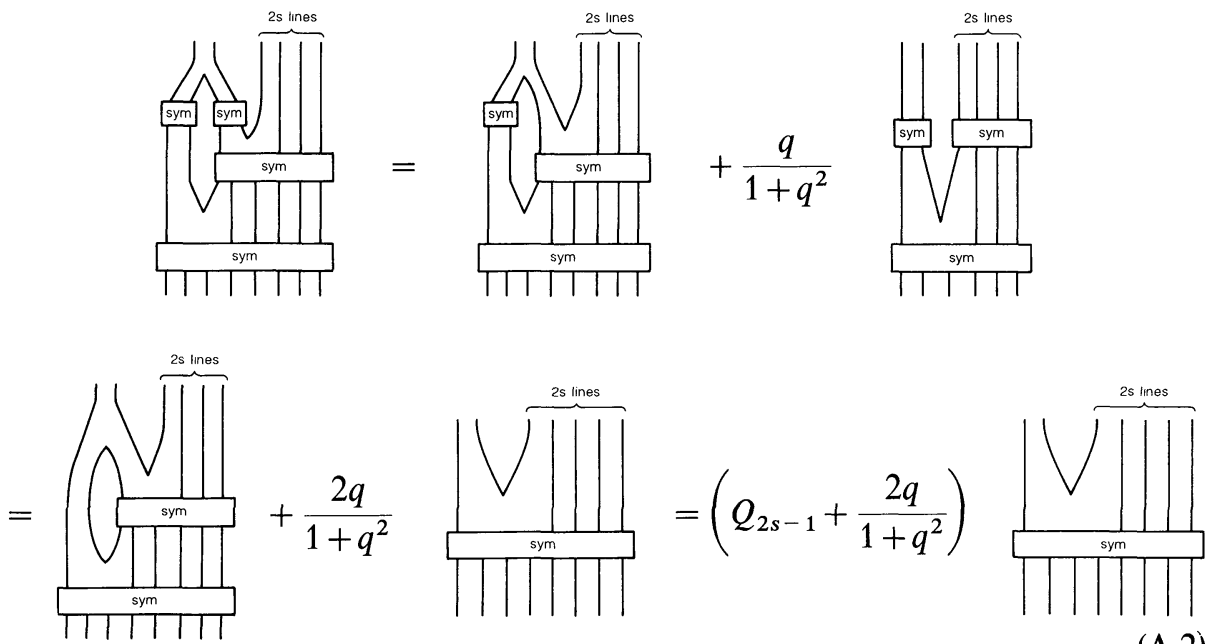

(A.2) 
where in the second equality we used the fact that on the symmetric tensors

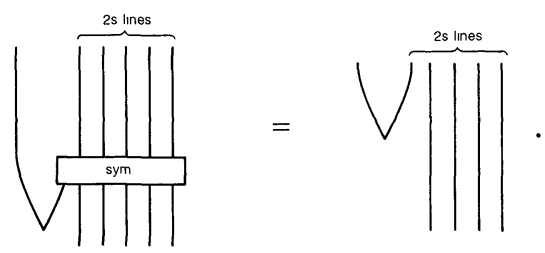

From (A.2) and (A.1) we obtain then

$$
\varrho_{s s s}=\left(Q_{2 s-1}+\frac{2 q}{1+q^{2}}\right)=-\frac{1+q^{4 s+2}}{q\left(1+q^{2}\right)[2 s]_{q}} .
$$

c) The Adjoint of Vector Operator. The computation of (2.48) is simple. We use the fact that the diagram for $\Psi_{s s^{\prime}}^{*}$ comes from the diagram for $\Psi_{s^{\prime} s}$ by reflecting it in a horizontal line. By (2.20), (2.21) we have $\left(E^{\prime}\right)^{*}=-\frac{1}{q} E$. This implies that symmetrization is unchanged by this operation since it uses a selfadjoint $\sigma$ [cf. (2.24)]. Taking this into account we get for example for $\omega_{(s+1) s}$ by (2.30) and (2.32),
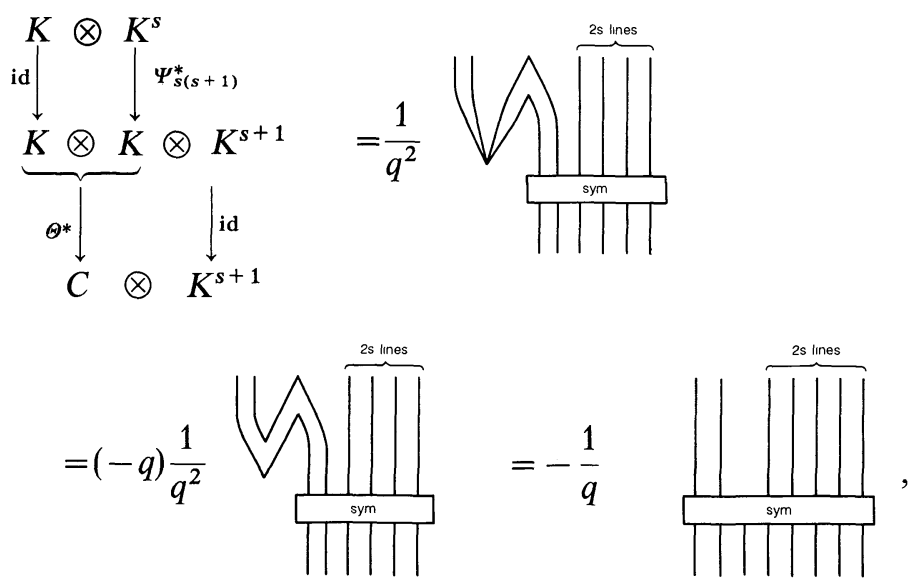

where we used property (2.22). From this by $(2.30) \omega_{(s+1) s}=-\frac{1}{q}$.

Acknowledgement. The author is very grateful to Prof. S. L. Woronowicz for helping him to understand the problems discussed here.

\section{References}

1. Gelfand, I.M., Ponomarev, V.A.: Indecomposable representations of the Lorentz group. In: Gelfand, I.M. (ed.) Collected papers, vol. II. Berlin, Heidelberg, New York: Springer 1988, p. 695

2. Gelfand, I.M., Yaglom, A.M.: General relativistically invariant equations and infinite dimensional representations of Lorentz group. In: Gelfand, I.M. (ed.) Collected papers, vol. II. Berlin, Heidelberg, New York: Springer 1988, p. 146 
3. Jimbo, M.: A $q$-difference analogue of $U(g)$ and the Yang-Baxter equation. Lett. Math. Phys. 10, 63-69 (1985)

4. Lassner, G.: Topological algebras of operators. Rep. Math. Phys. 3, 279-293 (1972)

5. Nalmark, M.A.: Linear representations of Lorentz group. Moscow: Gos. Izdat. Fiz-Mat. Literature, 1958 (in Russian)

6. Ogievetsky, O., Schmidke, W.B., Wess, J., Zumino, B.: Six generator $q$-deformed Lorentz algebra. Preprint

7. Podleś, P.: Quantum spheres. Lett. Math. Phys. 14, 193-202 (1987)

8. Podleś, P., Woronowicz, S.L.: Quantum deformation of Lorentz group. Commun. Math. Phys. 130, 381-431 (1990)

9. Pusz, W.: On the implementation of $S U(2)$ action in the irreducible representations of twisted canonical commutation relations. Lett. Math. Phys. 21, 59-67 (1991)

10. Schmidke, W.B., Wess, J., Zumino, B.: A q-deformed Lorentz algebra. Preprint

11. Sklyanin, E.K.: Funk. Anal. and Appl. 17, N$^{\circ} 4,34-48$ (1983)

12. Takeuchi, M.: Finite dimensional representations of the quantum Lorentz group. Preprint

13. Woronowicz, S.L.: Twisted $S U(2)$ group. An example of non-commutative differential calculus. Publ. RIMS Kyoto Univ. 23, 117-181 (1987)

14. Woronowicz, S.L.: Compact matrix pseudogroups. Commun. Math. Phys. 111, 613-665 (1987)

15. Woronowicz, S.L.: A remark on compact matrix quantum groups. Lett. Math. Phys. 21, 35-39 (1991)

16. Woronowicz, S.L.: Unbounded elements affiliated with $C^{*}$-algebras and non-compact quantum groups. Commun. Math. Phys. 136, 399-432 (1991)

17. Woronowicz, S.L.: Quantum E(2) group and its Pontryagin dual. Lett. Math. Phys. 23, 251-263 (1991)

18. Woronowicz, S.L., Zakrzewski, S.: Quantum Lorentz group having Gauss decomposition property. To appear in Publications of RIMS, Kyoto University

19. Woronowicz, S.L., Zakrzewski, S.: Quantum deformations of Lorentz group. Hopf *-algebra level. Preprint

Communicated by K. Gawedzki 